\title{
Excited bands and signature dependent electromagnetic decay properties in neutron-rich ${ }^{159,161,163} \mathrm{Dy}$
}

\author{
Andrea Jungclaus \\ Departamento de Fisica Teórica, Universidad Autónoma de Madrid, E-28049 Madrid, Spain \\ and Instituto de Estructura de la Materia, Consejo Superior de Investigaciones Científicas, E-28006 Madrid, Spain
}

B. Binder, A. Dietrich, T. Härtlein, H. Bauer, Ch. Gund, D. Pansegrau, and D. Schwalm Max-Planck-Institut für Kernphysik, D-69029 Heidelberg, Germany

D. Bazzacco, E. Farnea, S. Lunardi, C. Rossi-Alvarez, and C. Ur Dipartimento di Fisica dell' Universitá and INFN, Sezione di Padova, I-35131 Padova, Italy

G. de Angelis, A. Gadea, and D. R. Napoli

INFN, Laboratori Nazionali di Legnaro, I-35020 Legnaro, Italy

\author{
X. R. Zhou \\ Institute of Theoretical Physics, Chinese Academy of Sciences, Beijing 100080, People's Republic of China \\ and Department of Physics, Tsinghua University, Beijing 100084, People's Republic of China
}

\section{Y. Sun}

Department of Physics and Astronomy, University of Tennessee, Knoxville, Tennessee 37996

and Department of Physics, University of Notre Dame, Notre Dame, Indiana 46556

(Received 5 December 2002; published 12 March 2003)

\begin{abstract}
High-spin states of the neutron-rich odd nuclei ${ }^{159,161,163}$ Dy have been studied using the incomplete fusion reactions ${ }^{158,160} \mathrm{Gd}\left({ }^{7} \mathrm{Li},(p, d, t) \times n\right)$. In ${ }^{159} \mathrm{Dy}$, the band crossing in the $11 / 2^{-}$[505] band has been observed for the first time. Moreover, $11 E 1$ transitions connecting both signatures of the $3 / 2^{-}$[521] band to the $5 / 2^{+}[642]$ band have been observed in this nucleus; the deduced $B(E 1) / B(E 2)$ ratios as well as the $B(M 1) / B(E 2)$ ratios for transitions within the $3 / 2^{-}[521]$ band show a pronounced signature dependence. In ${ }^{161} \mathrm{Dy}$ and ${ }^{163} \mathrm{Dy}$, rotational bands have been extended to significantly higher spin values. In ${ }^{161} \mathrm{Dy}$, the sequences built on the neutron $5 / 2^{-}$[523] and $3 / 2^{-}$[521] states have been followed up to spin $49 / 2^{-}$and $33 / 2^{-}$, respectively, and in both cases upbends have been observed around $\hbar \omega \approx 0.26 \mathrm{MeV}$. In addition, a new band most probably built on the $11 / 2^{-}$[505] single-particle state has been identified in this isotope. In ${ }^{163} \mathrm{Dy}$, both the $5 / 2^{-}$[523] ground state band and the structure built on the $5 / 2^{+}$[642] neutron orbit have been extended up to the $45 / 2^{-}$and $49 / 2^{+}$ states, respectively. However, no band crossing has been observed in this nucleus. The properties of the observed bands in ${ }^{159,161,163}$ Dy are discussed and compared to calculations performed within the projected shell model.
\end{abstract}

DOI: 10.1103/PhysRevC.67.034302

PACS number(s): 23.20.Lv, 27.70.+q, 21.10.Re

\section{INTRODUCTION}

The odd Dy isotopes investigated in the present work belong to the region of deformed rare-earth nuclei in which pioneering work about the irregularities of the rotational motion, especially the backbending phenomenon [1], has been performed in the 1970s [2,3]. The comparison of rotational bands based on different single-particle orbits in a number of odd- $N$ and odd- $Z$ nuclei led to the conclusion that the alignment of only one single high- $j$ nucleon pair (in the rare-earth region a $i_{13 / 2}$ neutron pair) is responsible for the backbending process, i.e., that the crossing of the ground state band with a band built on a broken $i_{13 / 2}$ neutron pair causes the backbending observed in the Yrast sequence. A lot of experimental information about high-spin states is now available for the neutron deficient nuclei in this region. However, for the neutron rich isotopes, which cannot be populated in heavy-ion induced fusion-evaporation reactions, the available informa- tion is still rather meager. In this paper we report on a study of ${ }^{159,161,163}$ Dy using incomplete fusion reactions. Rotational bands in all three isotopes could be extended to considerably higher spin states than known before. Concerning the question of octupole softness in odd- $A$ nuclei, the importance of information on the angular momentum dependence of the $E 1$ transition strength between bands built on different configurations was pointed out in a series of papers [4-6]. We therefore searched for such $E 1$ transitions and found them in all three isotopes under study. The experimental details are given in Sec. II followed by the presentation of the results in Sec. III. The observed behavior of the rotational bands as well as their electromagnetic decay properties, namely the $B(E 1) / B(E 2)$ and $B(M 1) / B(E 2)$ ratios, are discussed in Sec. IV. Finally, the experimental results are compared to calculations performed within the projected shell model in Sec. V and the paper is closed by concluding remarks. 


\section{EXPERIMENTS}

To populate excited states in the nuclei ${ }^{159,161,163} \mathrm{Dy}$, the incomplete fusion reactions ${ }^{158,160} \mathrm{Gd}\left({ }^{7} \mathrm{Li},(p, d, t) \times n\right)$ at the beam energy of $56 \mathrm{MeV}$ were employed. The beam was delivered by the XTU tandem accelerator of the Laboratori Nazionali di Legnaro and directed onto targets with thicknesses of $3.7 \mathrm{mg} / \mathrm{cm}^{2}\left({ }^{158} \mathrm{Gd}\right)$ and $3.9 \mathrm{mg} / \mathrm{cm}^{2}\left({ }^{160} \mathrm{Gd}\right)$, respectively. The $\gamma$ radiation was detected in the 40 Comptonsuppressed Ge detectors of the GASP array and the 80element BGO inner ball. In addition, charged particles were detected in the Si ball ISIS consisting of $40 \mathrm{Si} \Delta E-E$ telescopes arranged in the same geometry as the Ge crystals in GASP, namely in seven rings with $\Theta=35^{\circ}, 59^{\circ}, 72^{\circ}$, $90^{\circ}, 108^{\circ}, 121^{\circ}$, and $145^{\circ}$ with respect to the beam. To protect the $\mathrm{Si}$ detectors from damage by scattered beam particles, an absorber tube consisting of $100-\mu \mathrm{m} \mathrm{Cu}$ and $12-\mu \mathrm{m}$ $\mathrm{Al}$ was mounted around the beam axis. All events with at least three coincident $\gamma$ rays in the Ge detectors or two $\gamma$ in the Ge plus one particle detected in the Si ball were recorded on tape with the additional condition that the $\gamma$ multiplicity in the BGO ball was 3 or higher. More details about this experiment are given in Ref. [7].

\section{DATA ANALYSIS AND RESULTS}

In the reactions ${ }^{158,160} \mathrm{Gd}\left({ }^{7} \mathrm{Li},(p, d, t) \times n\right)$, a number of Ho and Dy isotopes are produced. Whereas the Ho nuclei are populated after the emission of 4-7 neutrons, the emission of a charged particle, i.e., either a proton, a deuteron, or a triton, goes along with the population of the Dy isotopes. As discussed in detail in Ref. [7], the reaction mechanism responsible for the observed high cross sections for the charged particle channels is incomplete fusion. One advantage of this type of reaction is that the different final products are populated with different probabilities via the channels $p x n, d(x-1) n$ and $t(x-2) n$. For the lighter isotopes, the triton channel is most probable whereas for the heavier ones the proton channel is the most important one. This property together with the charged particle identification capability of the ISIS array allowed us to produce rather clean $\gamma \gamma$ matrices for each of the three isotopes ${ }^{159,161,163}$ Dy under study. In the following subsections, the data analysis and the resulting extensions of the level schemes will be discussed for each of the isotopes separately.

\section{A. Level scheme of ${ }^{159} \mathrm{Dy}$}

Previous work on ${ }^{159}$ Dy identified three rotational bands based on the $3 / 2^{-}[521], 5 / 2^{+}[642]$, and $11 / 2^{-}$[505] neutron single-particle states, which were observed up to the $21 / 2^{-}, 45 / 2^{+}$, and $23 / 2^{-}$states, respectively [8-10]. More recently, using the EUROBALL IV spectrometer and deepinelastic reactions, Liang et al. observed the $\alpha=+1 / 2$ sequence of the $5 / 2^{+}[642]$ band up to $61 / 2^{+}$[11], and Sugawara et al. succeeded to extend the $3 / 2^{-}[521]$ and the $11 / 2^{-}$[505] bands to spin $57 / 2^{-}$and $29 / 2^{-}$, respectively, using the ${ }^{150} \mathrm{Nd}\left({ }^{13} \mathrm{C}, 4 n\right)$ reaction $[12]$.

In our experiment using a ${ }^{158} \mathrm{Gd}$ target, ${ }^{159} \mathrm{Dy}$ is the second strongest Dy isotope produced behind ${ }^{160} \mathrm{Dy}$. The more

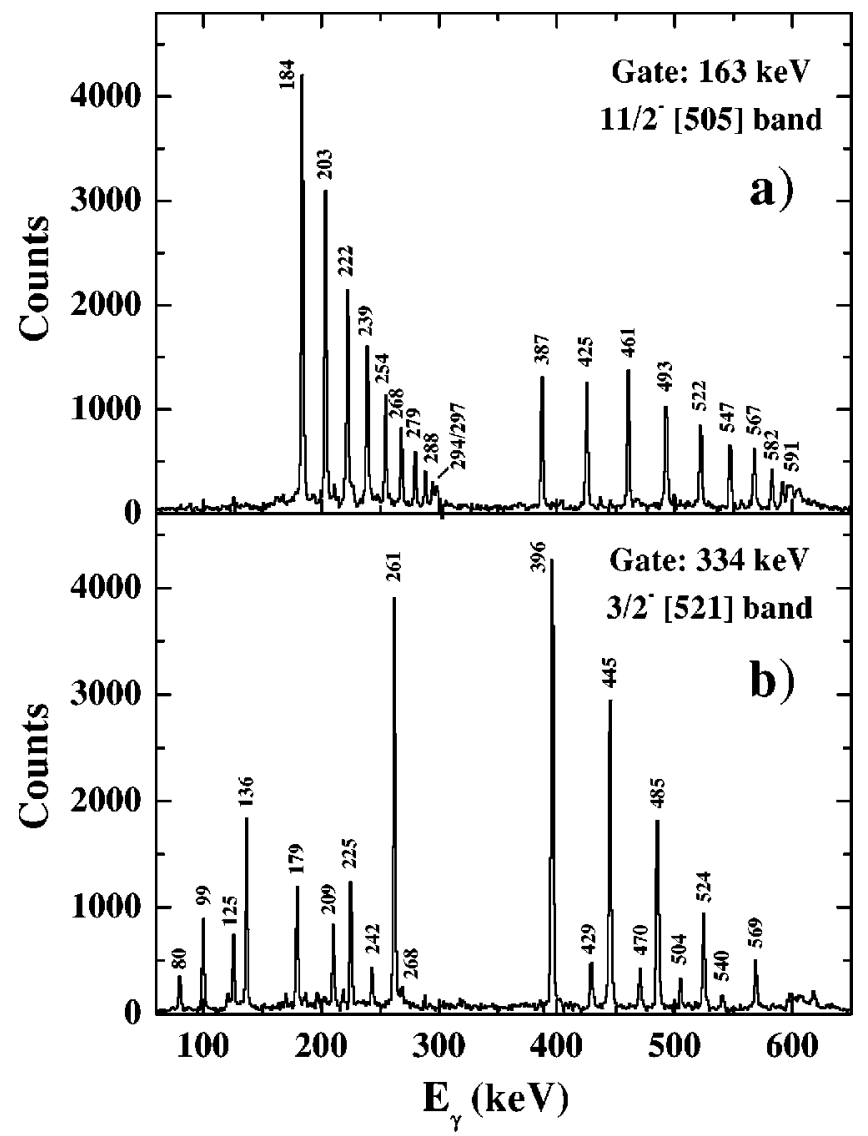

FIG. 1. $\gamma \gamma$ coincidence spectra in ${ }^{159} \mathrm{Dy}$ with a gate on (a) the $163-\mathrm{keV} 13 / 2^{-} \rightarrow 11 / 2^{-}$member of the $11 / 2^{-}$[505] band and (b) the $334-\mathrm{keV} 17 / 2^{-} \rightarrow 13 / 2^{-}$transition within the $3 / 2^{-}$[521] band.

detailed analysis of the reaction mechanism in Ref. [7] has shown that the ${ }^{158} \mathrm{Gd}\left({ }^{7} \mathrm{Li}, t 3 n\right)$ channel is the dominant one leading to the production of ${ }^{159} \mathrm{Dy}$, and the coincidence requirement with a triton detected in ISIS was therefore most important in preparing the ${ }^{159} \mathrm{Dy}$ data. To further reduce the main contamination by ${ }^{160} \mathrm{Dy}$, a $\gamma \gamma$ matrix produced in coincidence with high energy tritons was subtracted from a matrix produced in coincidence with low energy tritons which led to a rather clean ${ }^{159}$ Dy matrix. In Fig. 1, two coincidence spectra produced from this matrix are shown. In Fig. 1(a), a gate is set on the $163-\mathrm{keV} 13 / 2^{-} \rightarrow 11 / 2^{-}$member of the $11 / 2^{-}$[505] band, and Fig. 1(b) displays a spectrum with a gate on the $334-\mathrm{keV} 17 / 2^{-} \rightarrow 13 / 2^{-}$transition in the $3 / 2^{-}$[521] ground state band. The level scheme obtained in the present work is shown in Fig. 2. In comparison to Ref. [12], we were able to extend the $11 / 2^{-}$[505] band up to the $35 / 2^{-}$state. Moreover, E1 transitions have been observed over a wide spin range connecting both signatures of the $3 / 2^{-}[521]$ band to the $5 / 2^{+}[642]$ band. The intensities and assignments of all $\gamma$ rays observed in ${ }^{159}$ Dy are given in Table I.

\section{B. Level scheme of ${ }^{161} \mathrm{Dy}$}

In ${ }^{161} \mathrm{Dy}$, three rotational bands based on the $5 / 2^{+}$[642], the $5 / 2^{-}$[523], and the $3 / 2^{-}$[521] neutron single-particle orbits were known up to spin $33 / 2^{+}, 13 / 2^{-}$, and $9 / 2^{-}$, respec- 


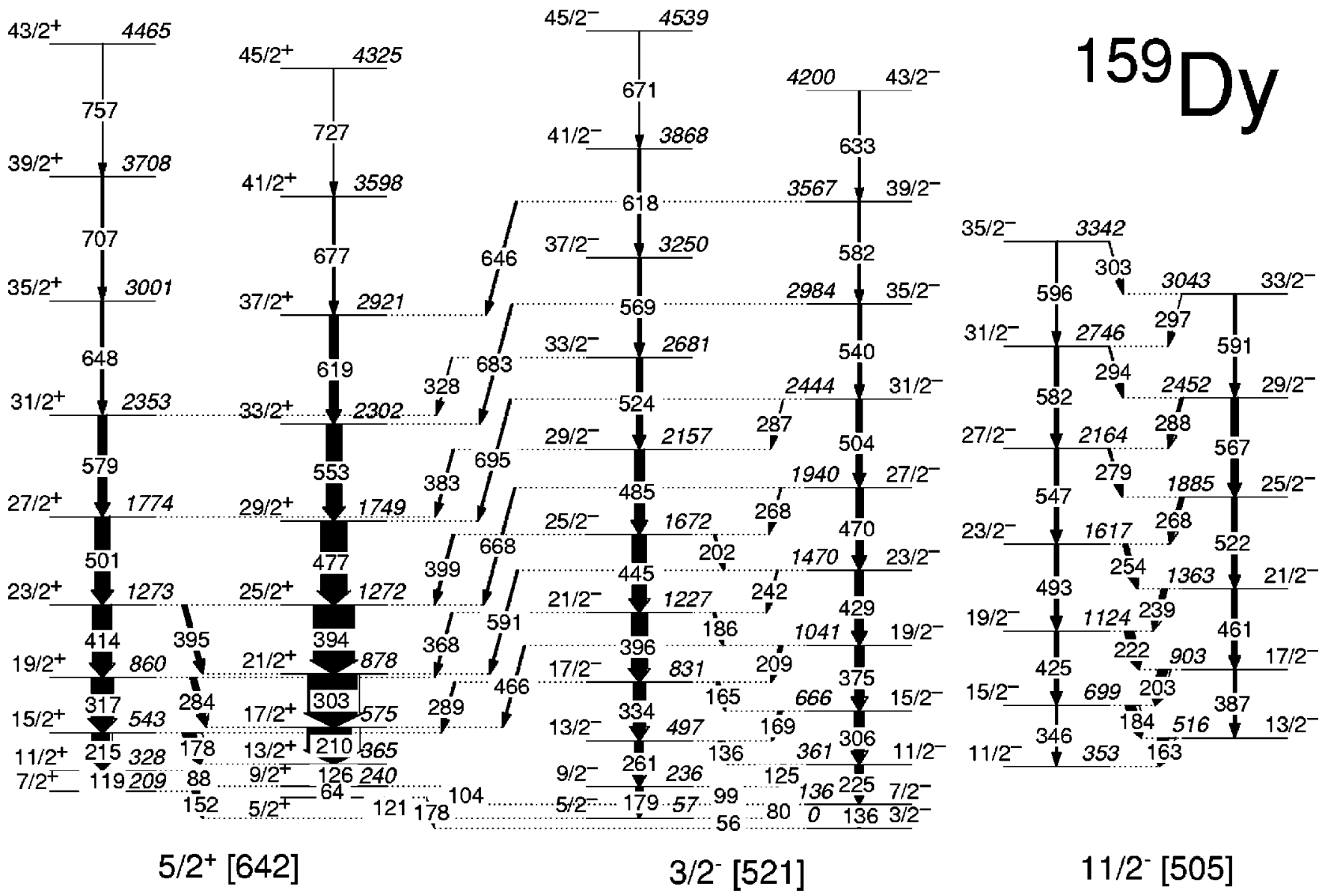

FIG. 2. The level scheme of ${ }^{159}$ Dy as obtained in the present work.

tively, from a study using the ${ }^{160} \mathrm{Gd}(\alpha, 3 n)$ reaction by Hjorth et al. [13]. In Ref. [14], the 3/2- [521] band was extended up to spin $13 / 2^{-}$using inelastic neutron scattering and in the same work an $11 / 2^{-}$state at $486 \mathrm{keV}$ was observed and interpreted as being based on the $11 / 2^{-}$[505] neutron orbit.

To study this nucleus, both data sets obtained with the ${ }^{158} \mathrm{Gd}$ and ${ }^{160} \mathrm{Gd}$ targets have been used. As ${ }^{161} \mathrm{Dy}$ is most strongly populated via the $t 3 n$ channel in the ${ }^{7} \mathrm{Li}+{ }^{160} \mathrm{Gd}$ reaction (compare Fig. 6 in Ref. [7]), a $\gamma \gamma$ matrix was sorted from this experiment with the requirement of a triton being detected in coincidence in the ISIS ball. The strongest contaminating channels in this matrix are the even neighbors ${ }^{162}$ Dy and ${ }^{160}$ Dy populated via the $t 2 n$ and $t 4 n$ channel, respectively. In order to reduce these contaminations, matrices in coincidence with high energetic deuterons obtained from both data sets have been subtracted from the triton gated matrix applying adequate normalization factors. Since these matrices contain mainly $\gamma$ rays belonging to ${ }^{162} \mathrm{Dy}$ and ${ }^{160}$ Dy [7], the result of this procedure is a quite pure ${ }^{161} \mathrm{Dy}$ matrix.

This matrix was then used to extend considerably the excitation scheme of this nucleus. The known rotational bands based on the $5 / 2^{+}[642], 5 / 2^{-}$[523], and 3/2 $[521]$ neutron single-particle states were observed in the present work up to spin $47 / 2^{+}, 49 / 2^{-}$, and $33 / 2^{-}$, respectively. To illustrate the quality of the data, three coincidence spectra are shown in Fig. 3. The coincidence spectra with gates on the 331- and 128-keV transitions in Figs. 3(a) and (b) illustrate the extensions of the known $5 / 2^{-}$[523] and $3 / 2^{-}$[521] bands. The states of both signatures of the $5 / 2^{-}$[523] band decay over a wide spin range via $E 1$ transitions to the $5 / 2^{+}$[642] ground state band. Moreover, a new band consisting of two sequences of $E 2$ and strong interconnecting $M 1$ transitions has been identified in ${ }^{161}$ Dy, which is displayed in Fig. 3(c). Although no $\gamma$ rays connecting this new structure to the known part of the level scheme were found, the band can be unequivocally assigned to ${ }^{161} \mathrm{Dy}$ on the basis of its observation and nonobservation under different particle conditions (see above). The most probable reason for the nonobservation of $\gamma$ rays from the decay of this band is an isomeric character of the band head. Taking into account our coincidence time window of about $70 \mathrm{~ns}$, no coincidences between populating and depopulating transitions are expected to be observed for state lifetimes above a few hundred nanoseconds. Another possible explanation for the nonobservation of $\gamma$ rays from the decay of this new band is that it decays directly to the ground state or to one of the low-lying states, e.g., the ones with excitation energies of 26 and $44 \mathrm{keV}$, the decays of which are not observed in the present experiment. In this case, however, the band should have been found already in the earlier studies concentrating on the low-spin part 
TABLE I. Energies and relative intensities of $\gamma$ transitions assigned to ${ }^{159} \mathrm{Dy}$. The Intensities are arbitrarily normalized to $I(210 \mathrm{keV})=1000$. The energy of the initial state $E_{i}$, the initial state spin $I_{i}$, and the final state spin $I_{f}$ are given for each transition in the last three columns.

\begin{tabular}{|c|c|c|c|c|}
\hline$E_{\gamma}(\mathrm{keV})^{\mathrm{a}}$ & $I_{\gamma}$ & $E_{i}(\mathrm{keV})$ & $I_{i}^{\pi}$ & $I_{f}^{\pi}$ \\
\hline 30.4 & & 240 & $9 / 2^{+}$ & $7 / 2^{+}$ \\
\hline 33.5 & & 209 & $7 / 2^{+}$ & $5 / 2^{+}$ \\
\hline 56.4 & & 57 & $5 / 2^{-}$ & $3 / 2^{-}$ \\
\hline 64.4 & & 240 & $9 / 2^{+}$ & $5 / 2^{+}$ \\
\hline 79.6 & 226(19) & 136 & $7 / 2^{-}$ & $5 / 2^{-}$ \\
\hline 88.2 & $475(32)$ & 328 & $11 / 2^{+}$ & $9 / 2^{+}$ \\
\hline 99.2 & $275(22)$ & 236 & $9 / 2^{-}$ & $7 / 2^{-}$ \\
\hline 102.7 & $162(15)$ & 240 & $9 / 2^{+}$ & $7 / 2^{-}$ \\
\hline 119.2 & $212(18)$ & 328 & $11 / 2^{+}$ & $7 / 2^{+}$ \\
\hline 120.8 & & 177 & $5 / 2^{+}$ & $5 / 2^{-}$ \\
\hline 125.0 & $117(12)$ & 361 & $11 / 2^{-}$ & $9 / 2^{-}$ \\
\hline 125.6 & $661(42)$ & 365 & $13 / 2^{+}$ & $9 / 2^{+}$ \\
\hline 135.8 & $122(13)$ & 497 & $13 / 2^{-}$ & $11 / 2^{-}$ \\
\hline 136.4 & $157(15)$ & 136 & $7 / 2^{-}$ & $3 / 2^{-}$ \\
\hline 152.2 & & 209 & $7 / 2^{+}$ & $5 / 2^{-}$ \\
\hline 162.5 & & 516 & $13 / 2^{-}$ & $11 / 2^{-}$ \\
\hline 165.0 & $69(9)$ & 831 & $17 / 2^{-}$ & $15 / 2^{-}$ \\
\hline 169.2 & $71(9)$ & 666 & $15 / 2^{-}$ & $13 / 2^{-}$ \\
\hline 177.5 & & 177 & $5 / 2^{+}$ & $3 / 2^{-}$ \\
\hline 177.7 & $365(26)$ & 543 & $15 / 2^{+}$ & $13 / 2^{+}$ \\
\hline 179.1 & $130(13)$ & 236 & $9 / 2^{-}$ & $5 / 2^{-}$ \\
\hline 183.5 & $260(21)$ & 699 & $15 / 2^{-}$ & $13 / 2^{-}$ \\
\hline 186.1 & $44(7)$ & 1227 & $21 / 2^{-}$ & $19 / 2^{-}$ \\
\hline 202.3 & & 1672 & $25 / 2^{-}$ & $23 / 2^{-}$ \\
\hline 203.3 & 228(19) & 903 & $17 / 2^{-}$ & $15 / 2^{-}$ \\
\hline 209.3 & $62(9)$ & 1041 & $19 / 2^{-}$ & $17 / 2^{-}$ \\
\hline 210.2 & $1000(59)$ & 575 & $17 / 2^{+}$ & $13 / 2^{+}$ \\
\hline 215.1 & $383(27)$ & 543 & $15 / 2^{+}$ & $11 / 2^{+}$ \\
\hline 221.7 & $168(15)$ & 1124 & $19 / 2^{-}$ & $17 / 2^{-}$ \\
\hline 224.5 & $148(14)$ & 361 & $11 / 2^{-}$ & $7 / 2^{-}$ \\
\hline 238.6 & $105(12)$ & 1363 & $21 / 2^{-}$ & $19 / 2^{-}$ \\
\hline 242.4 & $23(5)$ & 1470 & $23 / 2^{-}$ & $21 / 2^{-}$ \\
\hline 253.9 & $86(10)$ & 1617 & $23 / 2^{-}$ & $21 / 2^{-}$ \\
\hline 261.4 & $169(16)$ & 497 & $13 / 2^{-}$ & $9 / 2^{-}$ \\
\hline 267.6 & $77(10)$ & 1885 & $25 / 2^{-}$ & $23 / 2^{-}$ \\
\hline 267.8 & $15(4)$ & 1940 & $27 / 2^{-}$ & $25 / 2^{-}$ \\
\hline 279.0 & $34(6)$ & 2164 & $27 / 2^{-}$ & $25 / 2^{-}$ \\
\hline 284.3 & $109(12)$ & 860 & $19 / 2^{+}$ & $15 / 2^{+}$ \\
\hline 287.1 & & 2444 & $31 / 2^{-}$ & $29 / 2^{-}$ \\
\hline 287.9 & $53(8)$ & 2452 & $29 / 2^{-}$ & $27 / 2^{-}$ \\
\hline 288.6 & $20(5)$ & 831 & $17 / 2^{-}$ & $15 / 2^{+}$ \\
\hline 293.9 & & 2746 & $31 / 2^{-}$ & $29 / 2^{-}$ \\
\hline 297.1 & & 3043 & $33 / 2^{-}$ & $31 / 2^{-}$ \\
\hline 302.9 & $986(59)$ & 878 & $21 / 2^{+}$ & $17 / 2^{+}$ \\
\hline 303.2 & & 3342 & $35 / 2^{-}$ & $33 / 2^{-}$ \\
\hline 305.5 & 193(17) & 666 & $15 / 2^{-}$ & $11 / 2^{-}$ \\
\hline
\end{tabular}

TABLE I. (Continued).

\begin{tabular}{|c|c|c|c|c|}
\hline$E_{\gamma}(\mathrm{keV})^{\mathrm{a}}$ & $I_{\gamma}$ & $E_{i}(\mathrm{keV})$ & $I_{i}^{\pi}$ & $I_{f}^{\pi}$ \\
\hline 316.9 & $414(29)$ & 860 & $19 / 2^{+}$ & $15 / 2^{+}$ \\
\hline 327.9 & & 2681 & $33 / 2^{-}$ & $31 / 2^{+}$ \\
\hline 334.4 & 223(19) & 831 & $17 / 2^{-}$ & $13 / 2^{-}$ \\
\hline 346.2 & $36(6)$ & 699 & $15 / 2^{-}$ & $11 / 2^{-}$ \\
\hline 367.5 & $32(6)$ & 1227 & $21 / 2^{-}$ & $19 / 2^{+}$ \\
\hline 374.5 & $178(16)$ & 1041 & $19 / 2^{-}$ & $15 / 2^{-}$ \\
\hline 382.8 & $14(4)$ & 2157 & $29 / 2^{-}$ & $27 / 2^{+}$ \\
\hline 387.1 & $62(9)$ & 903 & $17 / 2^{-}$ & $13 / 2^{-}$ \\
\hline 393.6 & $772(48)$ & 1272 & $25 / 2^{+}$ & $21 / 2^{+}$ \\
\hline 395.1 & $85(10)$ & 1273 & $23 / 2^{+}$ & $21 / 2^{+}$ \\
\hline 395.7 & $289(22)$ & 1227 & $21 / 2^{-}$ & $17 / 2^{-}$ \\
\hline 398.7 & $37(6)$ & 1672 & $25 / 2^{-}$ & $23 / 2^{+}$ \\
\hline 413.5 & $361(26)$ & 1273 & $23 / 2^{+}$ & $19 / 2^{+}$ \\
\hline 425.1 & $82(10)$ & 1124 & $19 / 2^{-}$ & $15 / 2^{-}$ \\
\hline 428.9 & $168(15)$ & 1470 & $23 / 2^{-}$ & $19 / 2^{-}$ \\
\hline 444.8 & $274(22)$ & 1672 & $25 / 2^{-}$ & $21 / 2^{-}$ \\
\hline 460.5 & $93(11)$ & 1363 & $21 / 2^{-}$ & $17 / 2^{-}$ \\
\hline 465.7 & $27(5)$ & 1041 & $19 / 2^{-}$ & $17 / 2^{+}$ \\
\hline 470.1 & $135(13)$ & 1940 & $27 / 2^{-}$ & $23 / 2^{-}$ \\
\hline 477.3 & 499(34) & 1749 & $29 / 2^{+}$ & $25 / 2^{+}$ \\
\hline 485.1 & 177(16) & 2157 & $29 / 2^{-}$ & $25 / 2^{-}$ \\
\hline 492.7 & $79(10)$ & 1617 & $23 / 2^{-}$ & $19 / 2^{-}$ \\
\hline 501.1 & $280(22)$ & 1774 & $27 / 2^{+}$ & $23 / 2^{+}$ \\
\hline 504.4 & 111(12) & 2444 & $31 / 2^{-}$ & $27 / 2^{-}$ \\
\hline 521.6 & $87(10)$ & 1885 & $25 / 2^{-}$ & $21 / 2^{-}$ \\
\hline 524.1 & 107(12) & 2681 & $33 / 2^{-}$ & $29 / 2^{-}$ \\
\hline 539.9 & & 2984 & $35 / 2^{-}$ & $31 / 2^{-}$ \\
\hline 546.8 & $77(10)$ & 2164 & $27 / 2^{-}$ & $23 / 2^{-}$ \\
\hline 552.5 & $292(23)$ & 2302 & $33 / 2^{+}$ & $29 / 2^{+}$ \\
\hline 567.1 & 133(13) & 2452 & $29 / 2^{-}$ & $25 / 2^{-}$ \\
\hline 568.5 & $40(7)$ & 3250 & $37 / 2^{-}$ & $33 / 2^{-}$ \\
\hline 579.0 & $160(15)$ & 2353 & $31 / 2^{+}$ & $27 / 2^{+}$ \\
\hline 582.0 & $83(10)$ & 2746 & $31 / 2^{-}$ & $27 / 2^{-}$ \\
\hline 582.4 & & 3567 & $39 / 2^{-}$ & $35 / 2^{-}$ \\
\hline 591.1 & $60(8)$ & 3043 & $33 / 2^{-}$ & $29 / 2^{-}$ \\
\hline 591.4 & $38(6)$ & 1470 & $23 / 2^{-}$ & $21 / 2^{+}$ \\
\hline 595.7 & & 3342 & $35 / 2^{-}$ & $31 / 2^{-}$ \\
\hline 618.1 & & 3868 & $41 / 2^{-}$ & $37 / 2^{-}$ \\
\hline 619.3 & $158(15)$ & 2921 & $37 / 2^{+}$ & $33 / 2^{+}$ \\
\hline 633.4 & & 4200 & $43 / 2^{-}$ & $39 / 2^{-}$ \\
\hline 646.4 & & 3567 & $39 / 2^{-}$ & $37 / 2^{+}$ \\
\hline 647.7 & $50(8)$ & 3001 & $35 / 2^{+}$ & $31 / 2^{+}$ \\
\hline 668.1 & $45(7)$ & 1940 & $27 / 2^{-}$ & $25 / 2^{+}$ \\
\hline 670.8 & & 4539 & $45 / 2^{-}$ & $41 / 2^{-}$ \\
\hline 677.4 & $44(7)$ & 3598 & $41 / 2^{+}$ & $37 / 2^{+}$ \\
\hline 682.7 & & 2984 & $35 / 2^{-}$ & $33 / 2^{+}$ \\
\hline 695.4 & $38(6)$ & 2444 & $31 / 2^{-}$ & $29 / 2^{+}$ \\
\hline 707.2 & & 3708 & $39 / 2^{+}$ & $35 / 2^{+}$ \\
\hline 727.2 & & 4325 & $45 / 2^{+}$ & $41 / 2^{+}$ \\
\hline 757.3 & & 4465 & $43 / 2^{+}$ & $39 / 2^{+}$ \\
\hline
\end{tabular}




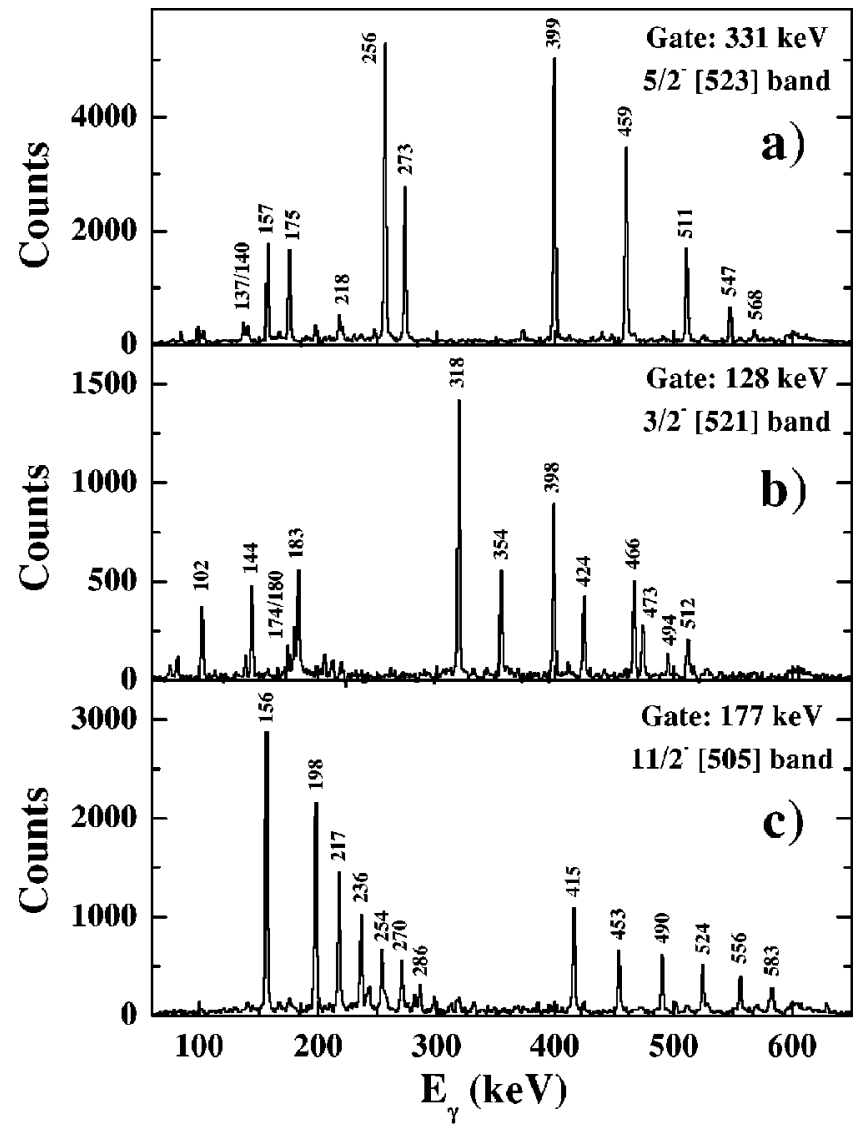

FIG. 3. $\gamma \gamma$ coincidence spectra in ${ }^{161}$ Dy with a gate on (a) the $331-\mathrm{keV} 17 / 2^{-} \rightarrow 13 / 2^{-}$transition within the $5 / 2^{-}$[523] band, (b) the $128-\mathrm{keV} 11 / 2^{-} \rightarrow 9 / 2^{-}$transition within the $3 / 2^{-}$[521] band, and (c) the $177-\mathrm{keV}\left(15 / 2^{-}\right) \rightarrow\left(13 / 2^{-}\right)$member of the proposed $11 / 2^{-}[505]$ band. of the level scheme $[13,14]$. Therefore, considering the similarity between this newly identified structure in ${ }^{161} \mathrm{Dy}$ and the $11 / 2^{-}$[505] band in ${ }^{159}$ Dy [compare Figs. 1(a) and 3(c) or Figs. 2 and 4] with a half-life of $\mathrm{T}_{1 / 2}=122(3) \mu s$ for the band head at $353 \mathrm{keV}$ [15], we propose that the new band is built on the $11 / 2^{-}$state at $486 \mathrm{keV}$ suggested in Ref. [14]. The extended level scheme obtained in the present work is shown in Fig. 4 and Table II summarizes the information about the observed $\gamma$ rays in ${ }^{161} \mathrm{Dy}$.

\section{Level scheme of ${ }^{163} \mathrm{Dy}$}

Two rotational bands were known in ${ }^{163}$ Dy prior to our work. The ground state band based on the $5 / 2^{-}$[523] neutron state was observed in Coulomb excitation up to spin $27 / 2^{-}$ [16] and a $5 / 2^{+}[642]$ band was identified in transfer reactions up to spin $13 / 2^{+}$[17]. Since this nucleus is mainly populated via the $p 3 n$ channel in the reaction ${ }^{7} \mathrm{Li}+{ }^{160} \mathrm{Gd}$ (see [7] for details), a $\gamma \gamma$ matrix in coincidence with high energetic protons was produced from this data set. However, in this matrix ${ }^{162} \mathrm{Dy}$ is still the dominant channel and it was therefore necessary to further clean it up by subtracting a matrix gated by high energetic deuterons, which contains mainly $\gamma$-rays from ${ }^{162} \mathrm{Dy}$. Using the resulting $\gamma \gamma$ matrix it was possible to considerably extend both known rotational bands in ${ }^{163} \mathrm{Dy}$. The ground state band was observed up to the $45 / 2^{-}$level and the $5 / 2^{+}$[642] band up to spin $49 / 2^{+}$. In the latter band, strong $M 1$ transitions from states of unfavored signature into the favored sequence were observed in analogy to the bands built on the same single-particle orbit in ${ }^{159,161}$ Dy. The newly proposed level scheme of this nucleus is shown in Fig. 5. For illustration, Fig. 6 shows the coincidence spectra obtained with a gate on (a) the $325-\mathrm{keV}$

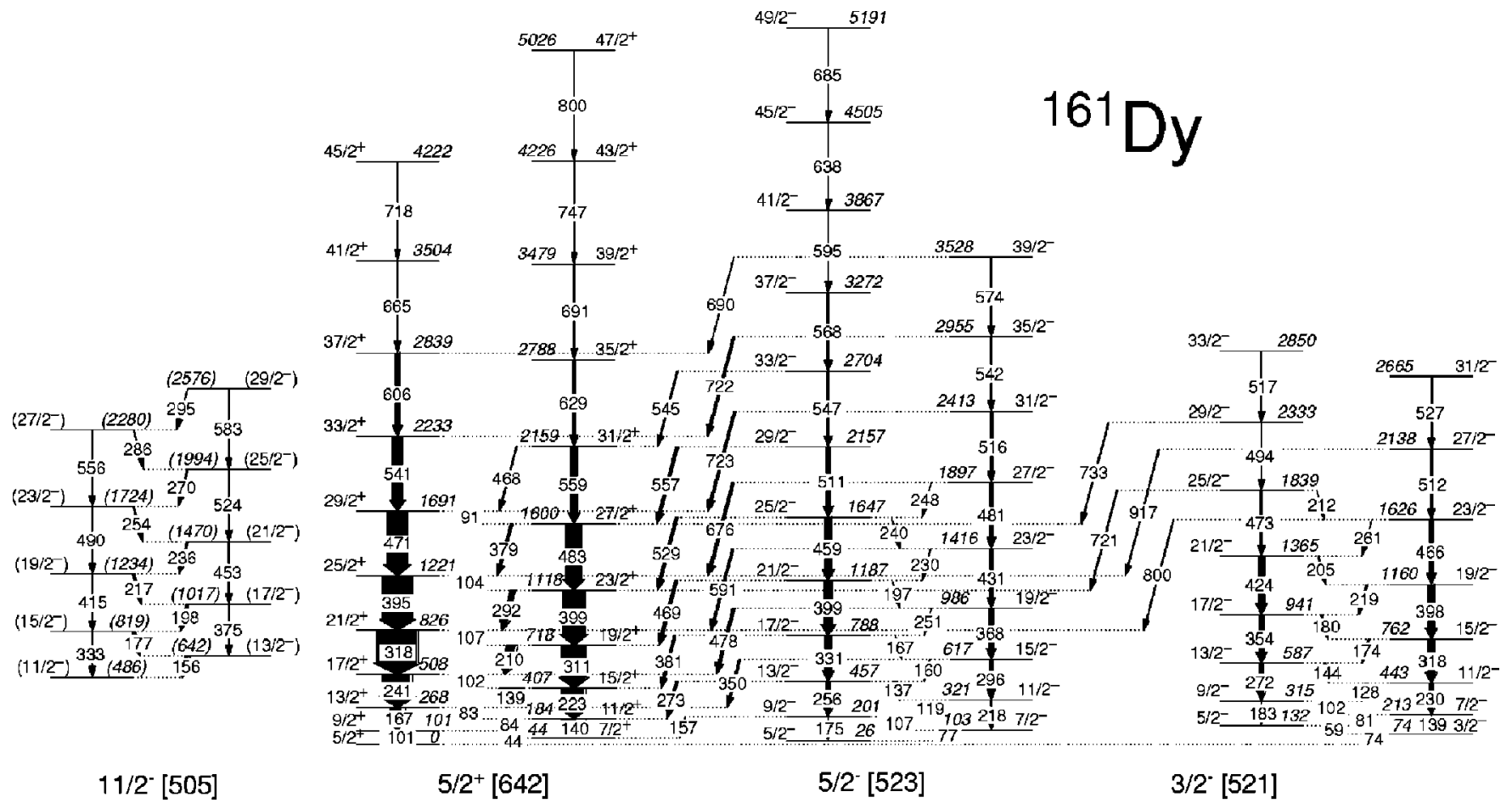

FIG. 4. The level scheme of ${ }^{161}$ Dy as obtained in the present work. 
TABLE II. Energies and relative intensities of $\gamma$ transitions assigned to ${ }^{161} \mathrm{Dy}$. The intensities are arbitrarily normalized to $I(318 \mathrm{keV})=1000$. The energy of the initial state $E_{i}$, the initial state spin $I_{i}$, and the final state spin $I_{f}$ are given for each transition in the last three columns.

\begin{tabular}{|c|c|c|c|c|c|c|c|c|c|}
\hline \multirow{3}{*}{\multicolumn{5}{|c|}{$\begin{array}{l}\text { signed to }{ }^{161} \text { Dy. The intensities are arbitrarily normalized to } \\
I(318 \mathrm{keV})=1000 \text {. The energy of the initial state } E_{i} \text {, the initial } \\
\text { state spin } I_{i} \text {, and the final state spin } I_{f} \text { are given for each transition } \\
\text { in the last three columns. }\end{array}$}} & $E_{\gamma}(\mathrm{keV})^{\mathrm{a}}$ & $I_{\gamma}$ & $E_{i}(\mathrm{keV})$ & $I_{i}^{\pi}$ & $I_{f}^{\pi}$ \\
\hline & & & & & \multirow{2}{*}{$\begin{array}{l}260.7 \\
270.4\end{array}$} & \multirow{3}{*}{$\begin{array}{c}20(5) \\
115(12)\end{array}$} & \multirow{2}{*}{$\begin{array}{c}1626 \\
(1994)\end{array}$} & \multirow{3}{*}{$\begin{array}{c}23 / 2^{-} \\
\left(25 / 2^{-}\right) \\
13 / 2^{-}\end{array}$} & \multirow{3}{*}{$\begin{array}{c}21 / 2^{-} \\
\left(23 / 2^{-}\right) \\
9 / 2^{-}\end{array}$} \\
\hline & & & & & & & & & \\
\hline$E_{\gamma}(\mathrm{keV})^{\mathrm{a}}$ & $I_{\gamma}$ & $E_{i}(\mathrm{keV})$ & $I_{i}^{\pi}$ & $I_{f}^{\pi}$ & 272.0 & & 587 & & \\
\hline 44 & & 44 & $7 / 2^{+}$ & $5 / 2^{+}$ & $\begin{array}{l}272.9 \\
285.5\end{array}$ & $\begin{array}{l}58(8) \\
13(4)\end{array}$ & $\begin{array}{c}457 \\
(2280)\end{array}$ & $13 / 2^{-}$ & $11 / 2^{+}$ \\
\hline 58.9 & & 132 & $5 / 2^{-}$ & $3 / 2^{-}$ & $\begin{array}{l}205.5 \\
291.7\end{array}$ & $127(13)$ & $\begin{array}{c}(2000) \\
1118\end{array}$ & $23 / 2^{+}$ & $\begin{array}{c}(25 / 2) \\
21 / 2^{+}\end{array}$ \\
\hline 74.4 & & 74 & $3 / 2^{-}$ & $5 / 2^{+}$ & 294.9 & $10(3)$ & (2576) & $\left(29 / 2^{-}\right)$ & $\left(27 / 2^{-}\right)$ \\
\hline 77.1 & & 103 & $7 / 2^{-}$ & $5 / 2^{-}$ & 296.5 & $83(10)$ & 617 & $15 / 2^{-}$ & $11 / 2^{-}$ \\
\hline 81.3 & & 213 & $7 / 2^{-}$ & $5 / 2^{-}$ & 311.3 & 597(39) & 718 & $19 / 2^{+}$ & $15 / 2^{+}$ \\
\hline 83.0 & & 268 & $13 / 2^{+}$ & $11 / 2^{+}$ & 317.8 & $1000(59)$ & 826 & $21 / 2^{+}$ & $17 / 2^{+}$ \\
\hline 83.8 & & 184 & $11 / 2^{+}$ & $9 / 2^{+}$ & 318.2 & $156(15)$ & 762 & $15 / 2^{-}$ & $11 / 2^{-}$ \\
\hline 91.2 & & 1691 & $29 / 2^{+}$ & $27 / 2^{+}$ & 330.8 & $138(14)$ & 788 & $17 / 2^{-}$ & $13 / 2^{-}$ \\
\hline 100.5 & & 101 & $9 / 2^{+}$ & $5 / 2^{+}$ & 333.5 & $26(5)$ & (819) & $\left(15 / 2^{-}\right)$ & $\left(11 / 2^{-}\right)$ \\
\hline 101.8 & $456(31)$ & 508 & $17 / 2^{+}$ & $15 / 2^{+}$ & 349.8 & $48(7)$ & 617 & $15 / 2^{-}$ & $13 / 2^{+}$ \\
\hline 102.1 & & 315 & $9 / 2^{-}$ & $7 / 2^{-}$ & 354.2 & $130(13)$ & 941 & $17 / 2^{-}$ & $13 / 2^{-}$ \\
\hline 103.6 & $42(7)$ & 1221 & $25 / 2^{+}$ & $23 / 2^{+}$ & 368.5 & $114(12)$ & 986 & $19 / 2^{-}$ & $15 / 2^{-}$ \\
\hline 106.7 & & 201 & $9 / 2^{-}$ & $7 / 2^{-}$ & 375.5 & $29(6)$ & (1017) & $\left(17 / 2^{-}\right)$ & $\left(13 / 2^{-}\right)$ \\
\hline 107.4 & $137(14)$ & 826 & $21 / 2^{+}$ & $19 / 2^{+}$ & 379.0 & $59(8)$ & 1600 & $27 / 2^{+}$ & $25 / 2^{+}$ \\
\hline 119.3 & & 321 & $11 / 2^{-}$ & $9 / 2^{-}$ & 381.0 & $53(8)$ & 788 & $17 / 2^{-}$ & $15 / 2^{+}$ \\
\hline 128.3 & $95(11)$ & 443 & $11 / 2^{-}$ & $9 / 2^{-}$ & 395.4 & $737(46)$ & 1221 & $25 / 2^{+}$ & $21 / 2^{+}$ \\
\hline 136.6 & & 457 & $13 / 2^{-}$ & $11 / 2^{-}$ & 398.4 & $152(15)$ & 1160 & $19 / 2^{-}$ & $15 / 2^{-}$ \\
\hline 138.5 & & 213 & $7 / 2^{-}$ & $3 / 2^{-}$ & 398.7 & 213(18) & 1187 & $21 / 2^{-}$ & $17 / 2^{-}$ \\
\hline 138.6 & $585(38)$ & 407 & $15 / 2^{+}$ & $13 / 2^{+}$ & 399.3 & $551(36)$ & 1118 & $23 / 2^{+}$ & $19 / 2^{+}$ \\
\hline 139.8 & & 184 & $11 / 2^{+}$ & $7 / 2^{+}$ & 415.3 & $36(6)$ & (1234) & $\left(19 / 2^{-}\right)$ & $\left(15 / 2^{-}\right)$ \\
\hline 143.5 & $66(9)$ & 587 & $13 / 2^{-}$ & $11 / 2^{-}$ & 424.1 & $145(14)$ & 1365 & $21 / 2^{-}$ & $17 / 2^{-}$ \\
\hline 156.0 & 131(13) & $(642)$ & $\left(13 / 2^{-}\right)$ & $\left(11 / 2^{-}\right)$ & 430.9 & $79(10)$ & 1416 & $23 / 2^{-}$ & $19 / 2^{-}$ \\
\hline 157.1 & & 201 & $9 / 2^{-}$ & $7 / 2^{+}$ & 453.4 & $31(6)$ & (1470) & $\left(21 / 2^{-}\right)$ & $\left(17 / 2^{-}\right)$ \\
\hline 159.8 & & 617 & $15 / 2^{-}$ & $13 / 2^{-}$ & 459.4 & $157(15)$ & 1647 & $25 / 2^{-}$ & $21 / 2^{-}$ \\
\hline 166.9 & $369(27)$ & 268 & $13 / 2^{+}$ & $9 / 2^{+}$ & 466.2 & $108(12)$ & 1626 & $23 / 2^{-}$ & $19 / 2^{-}$ \\
\hline 166.9 & & 788 & $17 / 2^{-}$ & $15 / 2^{-}$ & 467.5 & $23(5)$ & 2159 & $31 / 2^{+}$ & $29 / 2^{+}$ \\
\hline 174.5 & $42(7)$ & 762 & $15 / 2^{-}$ & $13 / 2^{-}$ & 468.8 & $69(9)$ & 1187 & $21 / 2^{-}$ & $19 / 2^{+}$ \\
\hline 175.3 & & 201 & $9 / 2^{-}$ & $5 / 2^{-}$ & 470.6 & $526(35)$ & 1691 & $29 / 2^{+}$ & $25 / 2^{+}$ \\
\hline 177.3 & $95(11)$ & (819) & $\left(15 / 2^{-}\right)$ & $\left(13 / 2^{-}\right)$ & 473.3 & & 1839 & $25 / 2^{-}$ & $21 / 2^{-}$ \\
\hline 179.6 & $36(6)$ & 941 & $17 / 2^{-}$ & $15 / 2^{-}$ & 477.7 & $66(9)$ & 986 & $19 / 2^{-}$ & $17 / 2^{+}$ \\
\hline 182.8 & & 315 & $9 / 2^{-}$ & $5 / 2^{-}$ & 480.8 & $91(11)$ & 1897 & $27 / 2^{-}$ & $23 / 2^{-}$ \\
\hline 196.7 & & 1187 & $21 / 2^{-}$ & $19 / 2^{-}$ & 482.6 & $394(28)$ & 1600 & $27 / 2^{+}$ & $23 / 2^{+}$ \\
\hline 197.8 & $73(9)$ & (1017) & $\left(17 / 2^{-}\right)$ & $\left(15 / 2^{-}\right)$ & 489.7 & $33(6)$ & (1724) & $\left(23 / 2^{-}\right)$ & $\left(19 / 2^{-}\right)$ \\
\hline 205.2 & $23(5)$ & 1365 & $21 / 2^{-}$ & $19 / 2^{-}$ & 494.5 & & 2333 & $29 / 2^{-}$ & $25 / 2^{-}$ \\
\hline 210.2 & $265(21)$ & 718 & $19 / 2^{+}$ & $17 / 2^{+}$ & 510.5 & & 2157 & $29 / 2^{-}$ & $25 / 2^{-}$ \\
\hline 211.9 & & 1839 & $25 / 2^{-}$ & $23 / 2^{-}$ & 512.1 & & 2138 & $27 / 2^{-}$ & $23 / 2^{-}$ \\
\hline 217.4 & $53(8)$ & (1234) & $\left(19 / 2^{-}\right)$ & $\left(17 / 2^{-}\right)$ & 516.0 & $50(8)$ & 2413 & $31 / 2^{-}$ & $27 / 2^{-}$ \\
\hline 217.5 & & 321 & $11 / 2^{-}$ & $7 / 2^{-}$ & 516.5 & & 2850 & $33 / 2^{-}$ & $29 / 2^{-}$ \\
\hline 218.7 & $29(6)$ & 1160 & $19 / 2^{-}$ & $17 / 2^{-}$ & 523.8 & $24(5)$ & (1994) & $\left(25 / 2^{-}\right)$ & $\left(21 / 2^{-}\right)$ \\
\hline 222.6 & 613(39) & 407 & $15 / 2^{+}$ & $11 / 2^{+}$ & 527.4 & & 2665 & $31 / 2^{-}$ & $27 / 2^{-}$ \\
\hline 230.0 & & 1416 & $23 / 2^{-}$ & $21 / 2^{-}$ & 528.7 & $63(9)$ & 1647 & $25 / 2^{-}$ & $23 / 2^{+}$ \\
\hline 230.3 & $108(12)$ & 443 & $11 / 2^{-}$ & $7 / 2^{-}$ & 541.0 & $252(20)$ & 2233 & $33 / 2^{+}$ & $29 / 2^{+}$ \\
\hline 235.9 & $36(6)$ & (1470) & $\left(21 / 2^{-}\right)$ & $\left(19 / 2^{-}\right)$ & 542.1 & & 2955 & $35 / 2^{-}$ & $31 / 2^{-}$ \\
\hline 239.8 & & 1647 & $25 / 2^{-}$ & $23 / 2^{-}$ & 544.9 & & 2704 & $33 / 2^{-}$ & $31 / 2^{+}$ \\
\hline 240.6 & $748(46)$ & 508 & $17 / 2^{+}$ & $13 / 2^{+}$ & 547.3 & & 2704 & $33 / 2^{-}$ & $29 / 2^{-}$ \\
\hline 247.8 & & 1897 & $27 / 2^{-}$ & $25 / 2^{-}$ & 555.8 & $20(5)$ & $(2280)$ & $\left(27 / 2^{-}\right)$ & $\left(23 / 2^{-}\right)$ \\
\hline 250.5 & & 986 & $19 / 2^{-}$ & $17 / 2^{-}$ & 556.8 & $67(9)$ & 2157 & $29 / 2^{-}$ & $27 / 2^{+}$ \\
\hline 253.7 & $24(5)$ & (1724) & $\left(23 / 2^{-}\right)$ & $\left(21 / 2^{-}\right)$ & 559.2 & 170(16) & 2159 & $31 / 2^{+}$ & $27 / 2^{+}$ \\
\hline 256.1 & $114(12)$ & 457 & $13 / 2^{-}$ & $9 / 2^{-}$ & 567.9 & & 3272 & $37 / 2^{-}$ & $33 / 2^{-}$ \\
\hline
\end{tabular}

TABLE II. (Continued). 
TABLE II. (Continued.)

\begin{tabular}{lcccc}
\hline \hline$E_{\gamma}(\mathrm{keV})^{\mathrm{a}}$ & $I_{\gamma}$ & $E_{i}(\mathrm{keV})$ & $I_{i}^{\pi}$ & $I_{f}^{\pi}$ \\
\hline 573.9 & & 3528 & $39 / 2^{-}$ & $35 / 2^{-}$ \\
583.0 & $14(4)$ & $(2576)$ & $\left(29 / 2^{-}\right)$ & $\left(25 / 2^{-}\right)$ \\
590.9 & $49(7)$ & 1416 & $23 / 2^{-}$ & $21 / 2^{+}$ \\
594.9 & & 3867 & $41 / 2^{-}$ & $37 / 2^{-}$ \\
606.1 & $126(13)$ & 2839 & $37 / 2^{+}$ & $33 / 2^{+}$ \\
628.8 & $69(9)$ & 2788 & $35 / 2^{+}$ & $31 / 2^{+}$ \\
638.4 & & 4505 & $45 / 2^{-}$ & $41 / 2^{-}$ \\
665.1 & $30(6)$ & 3504 & $41 / 2^{+}$ & $37 / 2^{+}$ \\
676.5 & $63(9)$ & 1897 & $27 / 2^{-}$ & $25 / 2^{+}$ \\
685.3 & & 5191 & $49 / 2^{-}$ & $45 / 2^{-}$ \\
689.6 & & 3528 & $39 / 2^{-}$ & $37 / 2^{+}$ \\
691.4 & $23(5)$ & 3479 & $39 / 2^{+}$ & $35 / 2^{+}$ \\
718.4 & $6(3)$ & 4222 & $45 / 2^{+}$ & $41 / 2^{+}$ \\
721.1 & & 1839 & $25 / 2^{-}$ & $23 / 2^{+}$ \\
721.5 & & 2955 & $35 / 2^{-}$ & $33 / 2^{+}$ \\
722.8 & & 2413 & $31 / 2^{-}$ & $29 / 2^{+}$ \\
733.0 & & 2333 & $29 / 2^{-}$ & $27 / 2^{+}$ \\
746.9 & $6(3)$ & 4226 & $43 / 2^{+}$ & $39 / 2^{+}$ \\
799.6 & & 5026 & $47 / 2^{+}$ & $43 / 2^{+}$ \\
\hline
\end{tabular}

${ }^{a}$ The uncertainties of the $\gamma$-ray energies are typically $0.1 \mathrm{keV}$ for $E_{\gamma}<1 \mathrm{MeV}$ and $0.2 \mathrm{keV}$ above.

$17 / 2^{-} \rightarrow 13 / 2^{-}$member of the ground state band and (b) the $515-\mathrm{keV} 33 / 2^{+} \rightarrow 29 / 2^{+}$transition within the $5 / 2^{+}[642]$ band. Finally, the intensities and assignments of all $\gamma$ rays observed in ${ }^{163}$ Dy are listed in Table III.

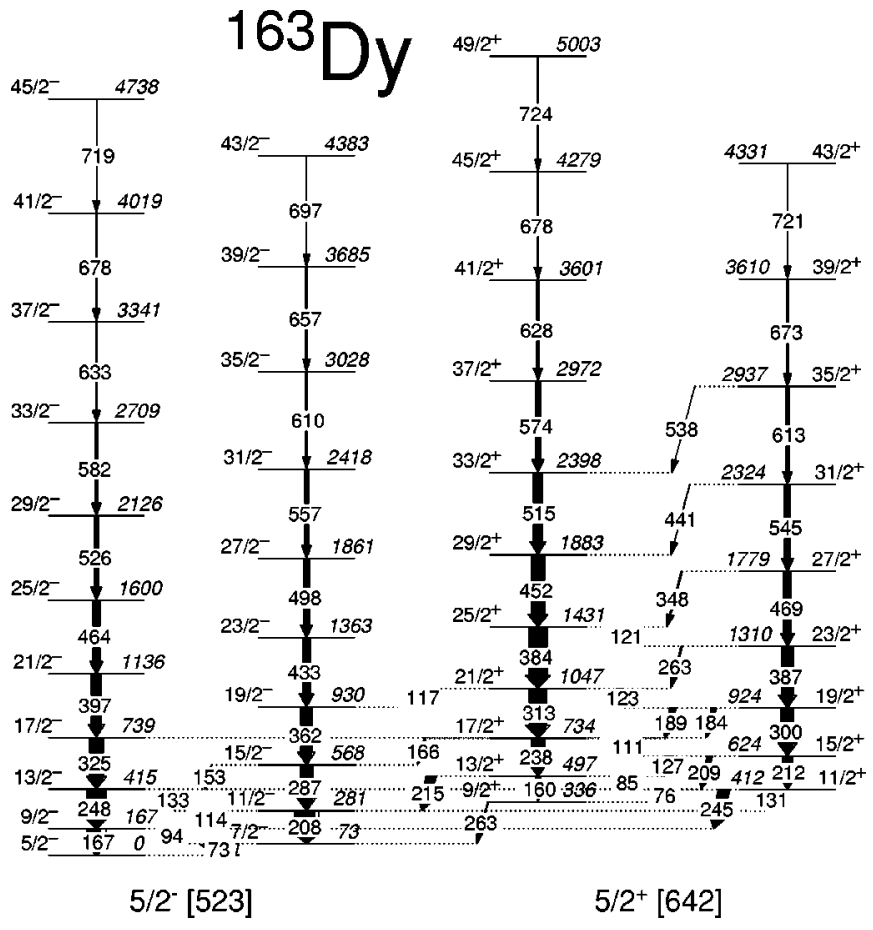

FIG. 5. The level scheme of ${ }^{163}$ Dy as obtained in the present work.

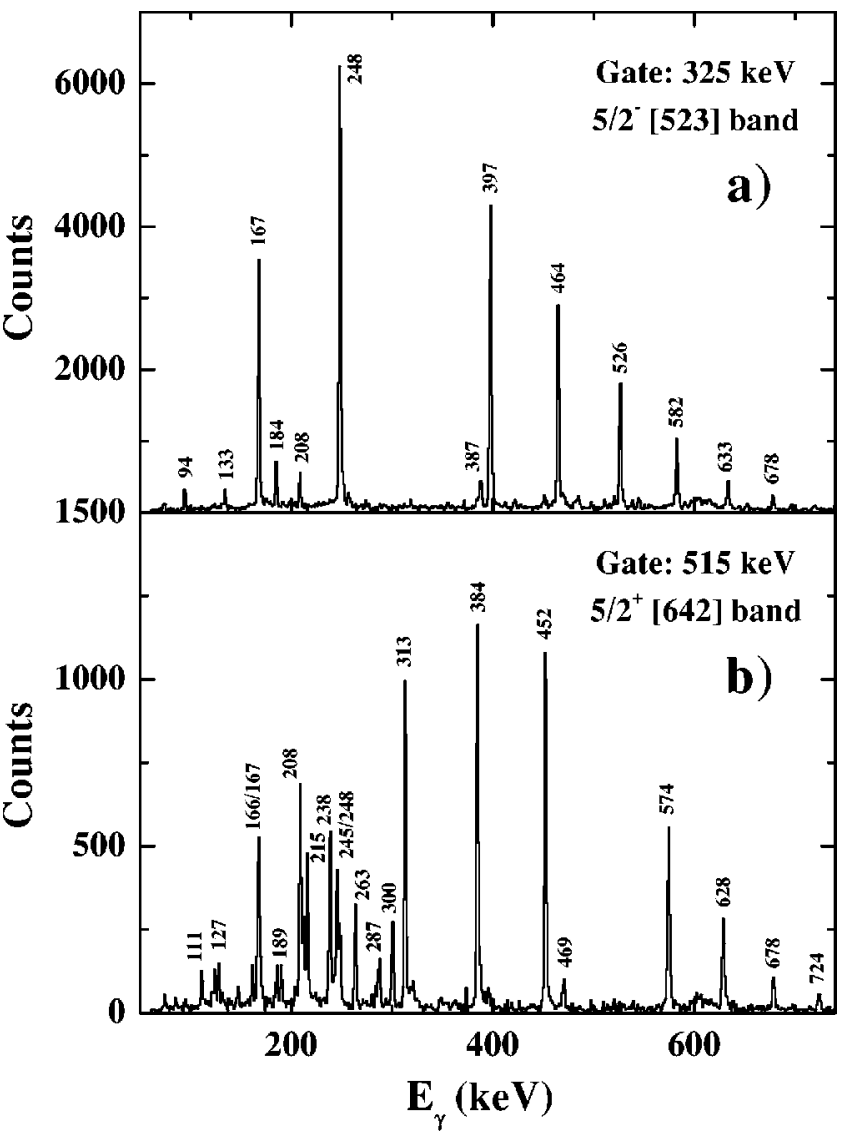

FIG. 6. $\gamma \gamma$ coincidence spectra in ${ }^{163} \mathrm{Dy}$ with a gate on (a) the $325-\mathrm{keV} 17 / 2^{-} \rightarrow 13 / 2^{-}$transition within the $5 / 2^{-}$[523] band and (b) the $515-\mathrm{keV} 33 / 2^{+} \rightarrow 29 / 2^{+}$member of the $5 / 2^{+}$[642] band.

\section{DISCUSSION}

As discussed in the previous section, several rotational bands in the odd ${ }^{159,161,163}$ Dy isotopes were extended up to significantly higher spin than known before and in ${ }^{161} \mathrm{Dy}$ a new rotational band was established. In order to discuss the properties of these bands at high spin, plots of the aligned angular momentum versus the rotational frequency $\hbar \omega$ are shown in Fig. 7 for all observed bands. For comparison the alignments for the yrast bands of the even neighbor isotopes $158,160,162$ Dy are shown in the figure, too. To determine the aligned angular momentum, the spin of a reference rotor with $I_{\text {ref }}=\left(\mathcal{J}_{0}+\mathcal{J}_{1} \omega^{2}\right)(\omega / \hbar)$ has been subtracted from the angular momentum $I$ as a function of $\omega$. The Harris parameters $\mathcal{J}_{0}=28 \hbar^{2} / \mathrm{MeV}$ and $\mathcal{J}_{1}=110 \hbar^{4} / \mathrm{MeV}^{3}$ have been used in accordance with Ref. [12].

For the $5 / 2^{+}[642] i_{13 / 2}$ band, which is the ground state band in ${ }^{161}$ Dy and which becomes the yrast band already at rather low rotational frequency in ${ }^{159}$ Dy and ${ }^{163} \mathrm{Dy}$, no band crossing was found in all three isotopes up to the highest frequencies ( $\hbar \omega \approx 0.37 \mathrm{MeV}$ ) observed in the present work. This is in accordance with the expectation since in this band an alignment of the $i_{13 / 2}$ neutrons is blocked by the odd neutron [2]. In the recent work on ${ }^{159}$ Dy by Sugawara et al. [12], a small alignment gain of about $2 \hbar$ has been observed in the $\alpha=+1 / 2$ sequence of this band at a higher frequency of $\hbar \omega \approx 0.4 \mathrm{MeV}$. 
TABLE III. Energies and relative intensities of $\gamma$ transitions assigned to ${ }^{163} \mathrm{Dy}$. The intensities are arbitrarely normalized to $I(208 \mathrm{keV})=1000$. The energy of the initial state $E_{i}$, the initial state spin $I_{i}$, and the final state spin $I_{f}$ are given for each transition in the last three columns.

\begin{tabular}{|c|c|c|c|c|}
\hline$E_{\gamma}(\mathrm{keV})^{\mathrm{a}}$ & $I_{\gamma}$ & $E_{i}(\mathrm{keV})$ & $I_{i}^{\pi}$ & $I_{f}^{\pi}$ \\
\hline 73.2 & & 73 & $7 / 2^{-}$ & $5 / 2^{-}$ \\
\hline 75.7 & & 412 & $11 / 2^{+}$ & $9 / 2^{+}$ \\
\hline 84.5 & & 497 & $13 / 2^{+}$ & $11 / 2^{+}$ \\
\hline 93.6 & & 167 & $9 / 2^{-}$ & $7 / 2^{-}$ \\
\hline 110.6 & $367(27)$ & 734 & $17 / 2^{+}$ & $15 / 2^{+}$ \\
\hline 114.0 & 181(16) & 281 & $11 / 2^{-}$ & $9 / 2^{-}$ \\
\hline 116.6 & $12(4)$ & 1047 & $21 / 2^{+}$ & $19 / 2^{-}$ \\
\hline 120.8 & $138(14)$ & 1431 & $25 / 2^{+}$ & $23 / 2^{+}$ \\
\hline 123.2 & $265(21)$ & 1047 & $21 / 2^{+}$ & $19 / 2^{+}$ \\
\hline 127.0 & $374(27)$ & 624 & $15 / 2^{+}$ & $13 / 2^{+}$ \\
\hline 130.6 & $114(12)$ & 412 & $11 / 2^{+}$ & $11 / 2^{-}$ \\
\hline 133.4 & $87(10)$ & 415 & $13 / 2^{-}$ & $11 / 2^{-}$ \\
\hline 153.4 & $25(5)$ & 568 & $15 / 2^{-}$ & $13 / 2^{-}$ \\
\hline 160.4 & 226(19) & 497 & $13 / 2^{+}$ & $9 / 2^{+}$ \\
\hline 165.9 & & 734 & $17 / 2^{+}$ & $15 / 2^{-}$ \\
\hline 167.2 & & 167 & $9 / 2^{-}$ & $5 / 2^{-}$ \\
\hline 184.4 & & 924 & $19 / 2^{+}$ & $17 / 2^{-}$ \\
\hline 189.3 & $251(20)$ & 924 & $19 / 2^{+}$ & $17 / 2^{+}$ \\
\hline 207.9 & $1000(59)$ & 281 & $11 / 2^{-}$ & $7 / 2^{-}$ \\
\hline 209.1 & 214(18) & 624 & $15 / 2^{+}$ & $13 / 2^{-}$ \\
\hline 211.8 & $408(29)$ & 624 & $15 / 2^{+}$ & $11 / 2^{+}$ \\
\hline 215.4 & $423(30)$ & 497 & $13 / 2^{+}$ & $11 / 2^{-}$ \\
\hline 237.7 & $535(35)$ & 734 & $17 / 2^{+}$ & $13 / 2^{+}$ \\
\hline 244.9 & $453(31)$ & 412 & $11 / 2^{+}$ & $9 / 2^{-}$ \\
\hline 247.7 & 798(49) & 415 & $13 / 2^{-}$ & $9 / 2^{-}$ \\
\hline 262.9 & & 336 & $9 / 2^{+}$ & $7 / 2^{-}$ \\
\hline 263.2 & & 1310 & $23 / 2^{+}$ & $21 / 2^{+}$ \\
\hline 287.0 & $528(35)$ & 568 & $15 / 2^{-}$ & $11 / 2^{-}$ \\
\hline 300.0 & $515(34)$ & 924 & $19 / 2^{+}$ & $15 / 2^{+}$ \\
\hline 312.6 & $736(46)$ & 1047 & $21 / 2^{+}$ & $17 / 2^{+}$ \\
\hline 324.5 & $563(37)$ & 739 & $17 / 2^{-}$ & $13 / 2^{-}$ \\
\hline 347.9 & $53(8)$ & 1779 & $27 / 2^{+}$ & $25 / 2^{+}$ \\
\hline 362.1 & $470(32)$ & 930 & $19 / 2^{-}$ & $15 / 2^{-}$ \\
\hline 384.1 & $769(47)$ & 1431 & $25 / 2^{+}$ & $21 / 2^{+}$ \\
\hline 386.6 & $492(33)$ & 1310 & $23 / 2^{+}$ & $19 / 2^{+}$ \\
\hline 397.0 & $406(29)$ & 1136 & $21 / 2^{-}$ & $17 / 2^{-}$ \\
\hline 432.6 & $333(25)$ & 1363 & $23 / 2^{-}$ & $19 / 2^{-}$ \\
\hline 441.2 & & 2324 & $31 / 2^{+}$ & $29 / 2^{+}$ \\
\hline 451.7 & $574(37)$ & 1883 & $29 / 2^{+}$ & $25 / 2^{+}$ \\
\hline 464.3 & $288(22)$ & 1600 & $25 / 2^{-}$ & $21 / 2^{-}$ \\
\hline 468.9 & $311(24)$ & 1779 & $27 / 2^{+}$ & $23 / 2^{+}$ \\
\hline 497.7 & 228(19) & 1861 & $27 / 2^{-}$ & $23 / 2^{-}$ \\
\hline 515.2 & $379(27)$ & 2398 & $33 / 2^{+}$ & $29 / 2^{+}$ \\
\hline 526.1 & 193(17) & 2126 & $29 / 2^{-}$ & $25 / 2^{-}$ \\
\hline 538.1 & & 2937 & $35 / 2^{+}$ & $33 / 2^{+}$ \\
\hline 544.8 & 236(19) & 2324 & $31 / 2^{+}$ & $27 / 2^{+}$ \\
\hline 557.1 & 131(13) & 2418 & $31 / 2^{-}$ & $27 / 2^{-}$ \\
\hline 574.1 & 222(19) & 2972 & $37 / 2^{+}$ & $33 / 2^{+}$ \\
\hline 582.3 & $113(12)$ & 2709 & $33 / 2^{-}$ & $29 / 2^{-}$ \\
\hline 610.4 & & 3028 & $35 / 2^{-}$ & $31 / 2^{-}$ \\
\hline
\end{tabular}

TABLE III. (Continued).

\begin{tabular}{lcccc}
\hline \hline$E_{\gamma}(\mathrm{keV})^{\mathrm{a}}$ & $I_{\gamma}$ & $E_{i}(\mathrm{keV})$ & $I_{i}^{\pi}$ & $I_{f}^{\pi}$ \\
\hline 613.3 & & 2937 & $35 / 2^{+}$ & $31 / 2^{+}$ \\
628.4 & $111(12)$ & 3601 & $41 / 2^{+}$ & $37 / 2^{+}$ \\
632.7 & $50(8)$ & 3341 & $37 / 2^{-}$ & $33 / 2^{-}$ \\
657.1 & $31(6)$ & 3685 & $39 / 2^{-}$ & $35 / 2^{-}$ \\
673.1 & $65(9)$ & 3610 & $39 / 2^{+}$ & $35 / 2^{+}$ \\
677.8 & $30(6)$ & 4019 & $41 / 2^{-}$ & $37 / 2^{-}$ \\
678.2 & & 4279 & $45 / 2^{+}$ & $41 / 2^{+}$ \\
697.4 & & 4383 & $43 / 2^{-}$ & $39 / 2^{-}$ \\
718.8 & & 4738 & $45 / 2^{-}$ & $41 / 2^{-}$ \\
721.2 & & 4331 & $43 / 2^{+}$ & $39 / 2^{+}$ \\
723.8 & & 5003 & $49 / 2^{+}$ & $45 / 2^{+}$ \\
\hline \hline
\end{tabular}

${ }^{\mathrm{a}}$ The uncertainties of the $\gamma$-ray energies are typically $0.1 \mathrm{keV}$ for $E_{\gamma}<1 \mathrm{MeV}$ and $0.2 \mathrm{keV}$ above.

In the ground state band in ${ }^{159} \mathrm{Dy}$, which is built on the $3 / 2^{-}$[521] $h_{9 / 2}$ configuration, no $i_{13 / 2}$ neutron blocking is involved and consequently a band crossing occurs for both signatures at a rotational frequency of $\hbar \omega \approx 0.25 \mathrm{MeV}$ [compare Fig. 7(a)]. In ${ }^{161}$ Dy this band shows a similar upbend at about the same frequency as in ${ }^{159} \mathrm{Dy}$. Here, however, the two sequences of different signature start to split up in the region of the band crossing. Such a pronounced splitting is not observed in the case of ${ }^{159} \mathrm{Dy}$.

The third Nilsson orbit close to the Fermi surface for the Dy isotopes under study is the $5 / 2^{-}[523] f_{7 / 2}$ state and a band built on this orbit forms the ground state band in ${ }^{163} \mathrm{Dy}$ and the negative parity yrast band in ${ }^{161} \mathrm{Dy}$. In the latter nucleus, this band behaves very similar to the $3 / 2^{-}[521]$ band, i.e., it shows a band crossing around $\hbar \omega \approx 0.26 \mathrm{MeV}$ and also in this band, a splitting between the two signature partners is observed in the band crossing region. In ${ }^{163} \mathrm{Dy}$, no irregularity is found in the $5 / 2^{-}$[523] band in the frequency region $\hbar \omega \leqslant 0.36 \mathrm{MeV}$ though a slight increase of the alignment is observed for frequency values larger than 0.30 $\mathrm{MeV}$. The origin of the different behavior of the $5 / 2^{-}[523] f_{7 / 2}$ band in the two neighboring isotopes, ${ }^{161} \mathrm{Dy}$ and ${ }^{163} \mathrm{Dy}$, is not obvious. As shown in Figs. 7(a) and (b), the band crossings in the $3 / 2^{-}$[521] and 5/2- [523] bands in ${ }^{159,161}$ Dy take place at a frequency about $40 \mathrm{keV}$ lower than the crossing frequency in the yrast band of the even Dy neighbor. This effect has been observed in a number of odd nuclei in the rare-earth region $[18,19]$ and is explained by a reduction of the neutron pairing correlations due to the"blocking" of the pairing contribution from a quasineutron orbit near the Fermi surface [18].

In the $11 / 2^{-}[505] h_{11 / 2}$ band in ${ }^{159}$ Dy, a band crossing was observed for the first time. As opposed to the $h_{9 / 2}$ and $f_{7 / 2}$ bands, this band shows an upbend at the same frequency as the yrast band in ${ }^{158} \mathrm{Dy}$. In ${ }^{161} \mathrm{Dy}$, the same band unfortunately has been observed only up to $\hbar \omega \approx 0.29 \mathrm{MeV}$ and up to this frequency no crossing was observed.

In all three odd Dy isotopes studied, a large energy splitting between the different signatures is observed for the positive parity yrast sequence in which the odd neutron occupies 


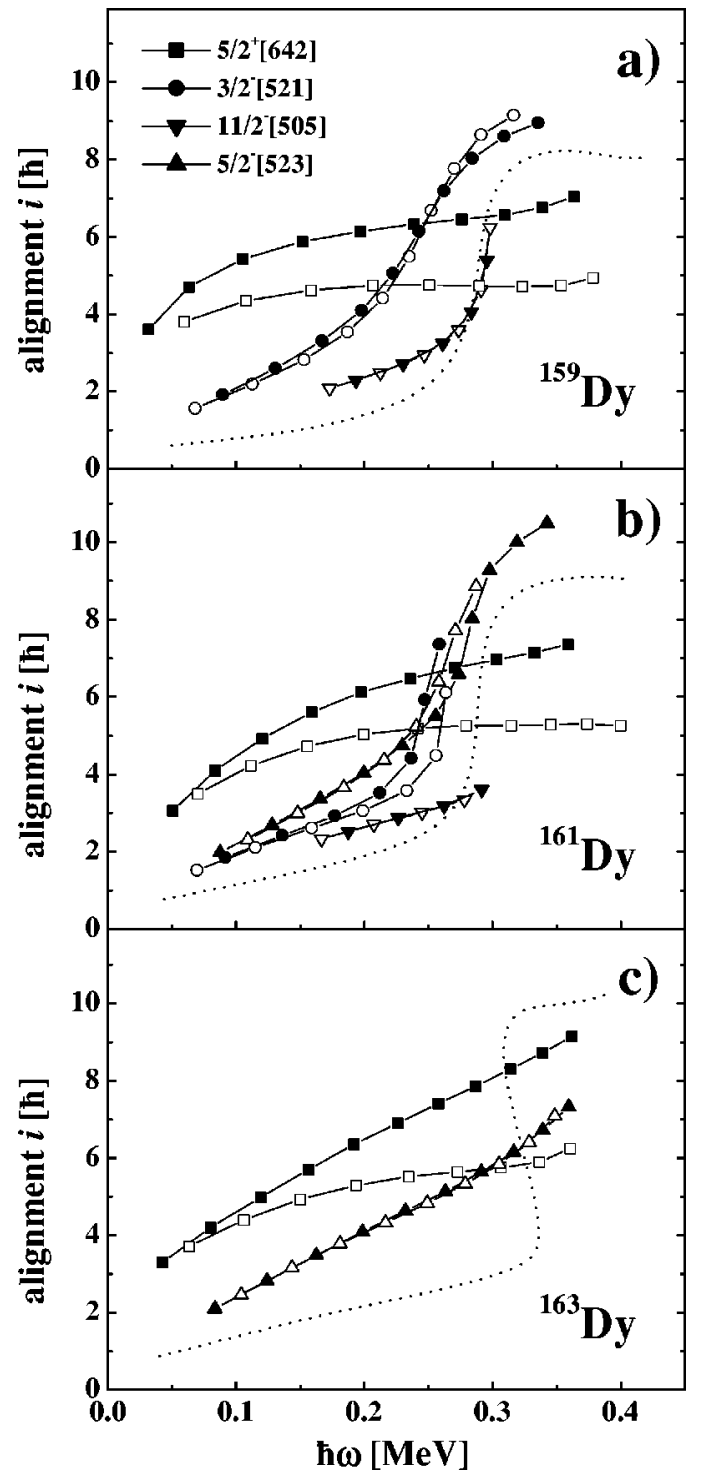

FIG. 7. Alignment plots for the rotational bands in ${ }^{159,161,163}$ Dy. States of signature $\alpha=+1 / 2$ are shown as filled symbols and the ones with $\alpha=-1 / 2$ with open symbols. The Harris parameters used for the reference rotor are $\mathcal{J}_{0}=28 \hbar^{2} / \mathrm{MeV}$ and $\mathcal{J}_{1}$ $=110 \hbar^{4} / \mathrm{MeV}^{3}$. The alignments of the yrast bands in the even neighbors ${ }^{158,160,162}$ Dy [in (a), (b), and (c), respectively] are shown for comparison by dotted lines.

the $5 / 2^{+}[642]$ Nilsson orbit. In Fig. 8(a), the quantity

$$
\begin{aligned}
S(I)= & E(I)-E(I-1) \\
& -\frac{1}{2}[E(I+1)-E(I)+E(I-1)-E(I-2)]
\end{aligned}
$$

is plotted as a function of the spin $I$. It can be read from this figure that for small and medium spin values, the energy splitting decreases slightly with increasing $N$ between ${ }^{159}$ Dy $(N=93)$ and ${ }^{163}$ Dy $(N=97)$. A similar trend has already been observed in the odd $\mathrm{Yb}$ isotopes from $N=91-95$ [19]. The energy splitting is a measure for the $\Omega=1 / 2$ component in the wave function. Since in this case the relevant

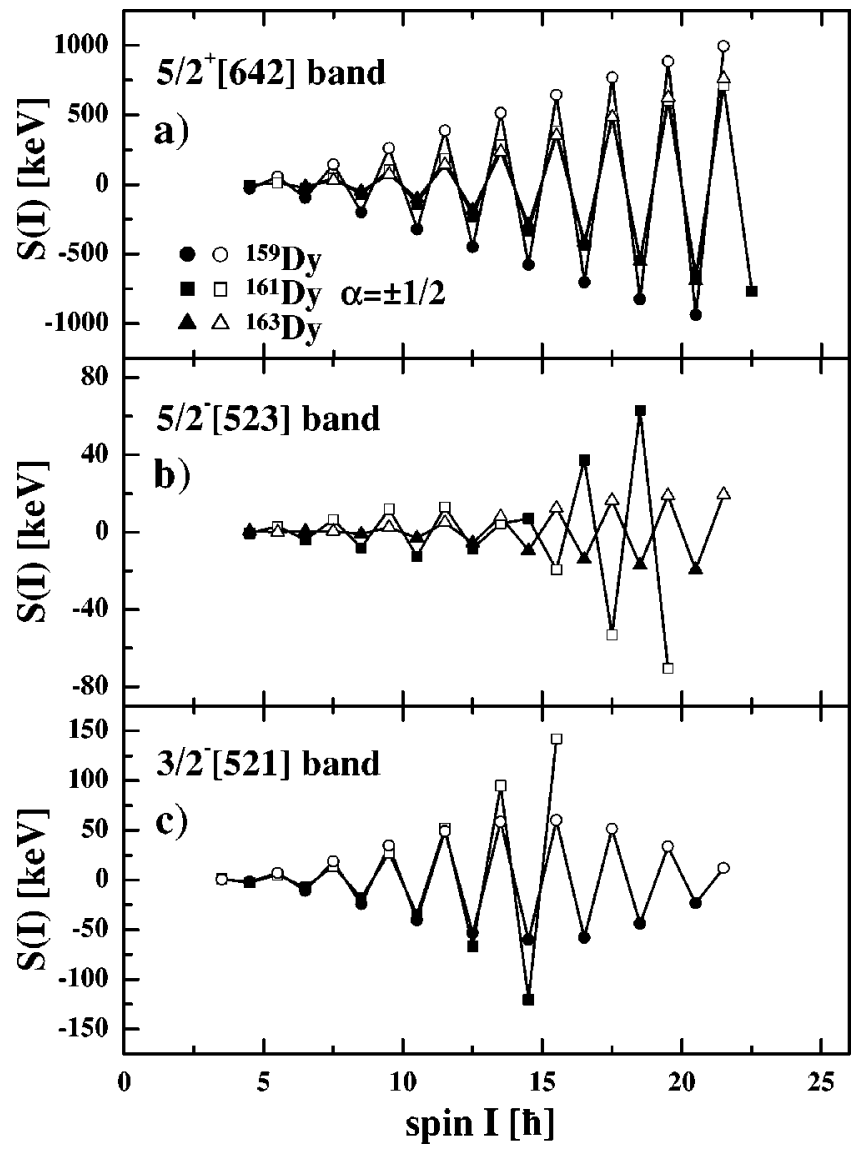

FIG. 8. Experimental energy differences $S(I)$ (see text for the definition) for the levels of (a) the $5 / 2^{+}[642]$, (b) the $5 / 2^{-}$[523], and (c) the $3 / 2^{-}[521]$ bands in ${ }^{159,161,163}$ Dy. $\alpha=+1 / 2(\alpha=-1 / 2)$ states are shown as filled (open) symbols.

$1 / 2^{+}[660]$ Nilsson orbit shifts away from the Fermi surface with increasing $N$, a decreasing splitting is to be expected.

It is also interesting to look for the signature splitting in bands based on nonunique parity orbitals. For the $5 / 2^{-}$[523] band, see Fig. 8(b), the splitting in ${ }^{161}$ Dy and ${ }^{163}$ Dy is, as expected, much smaller than for the $5 / 2^{+}[642]$ band. This behavior is, however, strongly nuclide dependent. Bands built on the same orbit display splittings as large as $300 \mathrm{keV}$ at $I$ values around $39 / 2$ for ${ }^{167} \mathrm{Yb}$ [20] and ${ }^{169} \mathrm{Hf}$ [21], which are $N=97$ isotones of ${ }^{163} \mathrm{Dy}$. On the other hand, the splitting of this band in the $N=95$ isotope ${ }^{163} \mathrm{Er}$ [22] is very similar to the one observed in its isotone ${ }^{161} \mathrm{Dy}$. Even the signature inversion present in ${ }^{161}$ Dy around spin $29 / 2$ is also found in ${ }^{163} \mathrm{Er}$ at about the same spin value. Concerning the $3 / 2^{-}[521]$ orbital, the splitting in the corresponding bands in ${ }^{159,161}$ Dy is again much smaller than for the $5 / 2^{+}[642]$ bands [see Fig. 8(c)]. An inversion of the splitting in the $3 / 2^{-}$[521] band in ${ }^{159}$ Dy was observed in Ref. [12] around spin 45/2.

An important aspect of the present experiment is the observation of large $E 1$ transition probabilities, large as compared to the single-particle expectations, as well as a signature dependence of these probabilities. Large $B(E 1)$ values have been associated [4-6] with the coupling of the odd nucleon to octupole vibrations. Octupole constrained Hartree-Fock-Bogoliubov calculations performed for the 
nucleus ${ }^{160}$ Dy with the Gogny force, see Ref. [7], have shown that the Dysprosium isotopes are indeed very soft in the octupole degree of freedom. Accordingly, we expect lowlying octupole vibrations, a strong coupling to the odd nucleon, and thus the occurence of large $B(E 1)$ values in these nuclei. A signature dependence of the $B(E 1)$ values has, to our knowledge, only been investigated experimentally in rare cases $[23,6]$. We actually succeeded to observe $E 1$ transitions over a wide spin range from the $3 / 2^{-}[521]$ to the $5 / 2^{+}[642]$ band in ${ }^{159}$ Dy and from the $5 / 2^{-}[523]$ to the $5 / 2^{+}[642]$ band in ${ }^{161}$ Dy. However, as no lifetimes were measured in the present work, only the ratios between the out-of-band $B(E 1 ; I \rightarrow I-1)$ values to the in-band $B(E 2 ; I \rightarrow I-2)$ values could be extracted from the measured intensity ratios without further assumptions using the relation

$$
\frac{B(E 1 ; I \rightarrow I-1)}{B(E 2 ; I \rightarrow I-2)}=0.7673 \times \frac{E_{\gamma}^{5}(E 2)}{E_{\gamma}^{3}(E 1)} \times \frac{I_{\gamma}(E 1)}{I_{\gamma}(E 2)}\left(10^{-6} \mathrm{fm}^{-2}\right)
$$

The intensity ratio $I_{\gamma}(E 1) / I_{\gamma}(E 2)$ for the decay of a given state $I$ has been determined in the most direct way, namely from the coincidence spectrum with a gate on the in-band transition populating the state under investigation. In this way systematical uncertainties are reduced at the expence of higher statistics.

Employing the generalized intensity relation (GIR) derived for the $B(E 1)$ values within the rotational model $[24,25]$, the $B(E 1) / B(E 2)$ ratios are expected to obey the relation

$$
\begin{aligned}
R(E 1 / E 2) \equiv & {\left[\frac{B\left(E 1 ; K_{i}, I \rightarrow K_{f}, I-1\right)}{B\left(E 2 ; K_{i}, I \rightarrow K_{i}, I-2\right)}\right]^{1 / 2} } \\
& \times\left|\frac{\left\langle I, K_{i}, 2,0 \mid I-2, K_{i}\right\rangle}{\left\langle I, K_{i}, 1,1 \mid I-1, K_{f}\right\rangle}\right| \\
= & \frac{1}{\sqrt{5 / 16 \pi} Q_{0}}\left[M_{1}-2 M_{2} \cdot I\right],
\end{aligned}
$$

with $M_{1}\left(2 M_{2} \cdot I\right)$ being the $I$-independent (leading order $I$-dependent) intrinsic $E 1$ matrix element and $Q_{0}$ the intrinsic quadrupole moment of the $K_{i}$ band. The experimental $R(E 1 / E 2)$ ratios are displayed as a function of $I$ in Fig. 9. Figure 9(a) shows the $R(E 1 / E 2)$ ratios for the decay of the $3 / 2^{-}$[521] band in ${ }^{159} \mathrm{Dy}$, Fig. 9(b) those for the decay of the $5 / 2^{-}$[523] band in ${ }^{161} \mathrm{Dy}$. In addition, the $R(E 1 / E 2)$ values obtained for the four $E 1$ transitions from the 3/2 $[521]$ to the $5 / 2^{+}[642]$ band in this nucleus are included, too. As expected we find large intrinsic $E 1$ matrix elements in both cases with a linear dependence on $I$ as predicted by the GIR [Eq. (3)]. However, a significant difference between the two bands is observed. The $R(E 1 / E 2)$ ratios for the decay of the $3 / 2^{-}[521]$ band in ${ }^{159}$ Dy exhibit a characteristic signature splitting, namely, the measured $B(E 1) / B(E 2)$ ratios for the decay of states with positive signature are systematically larger than the ones for the decay of negative signature

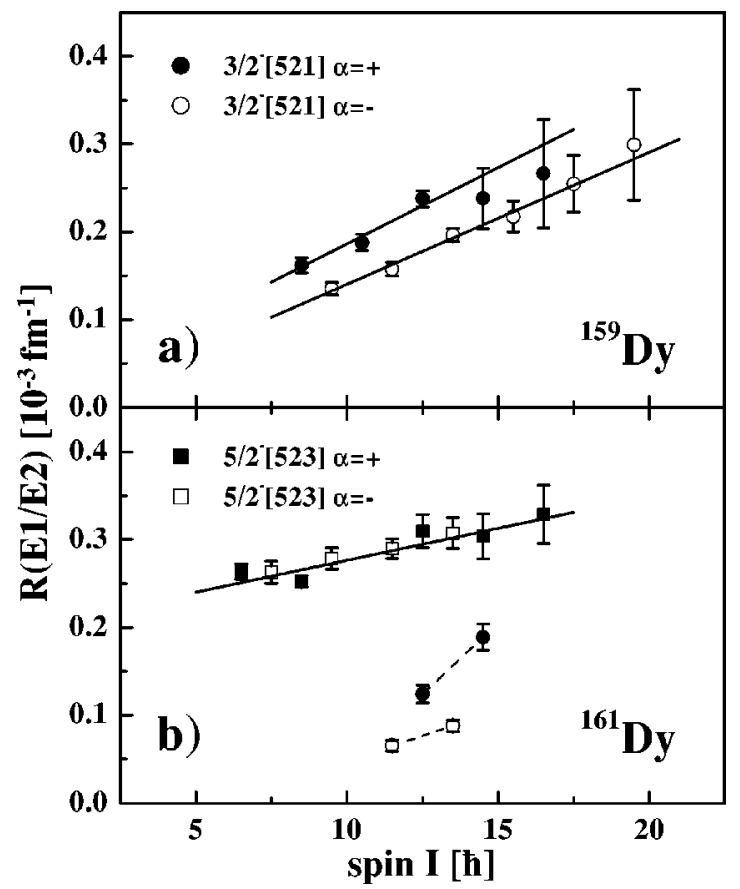

FIG. 9. Experimental $R(E 1 / E 2)$ ratios (see text for the definition) as a function of spin for (a) the $3 / 2^{-}$[521] band in ${ }^{159}$ Dy and (b) the $3 / 2^{-}[521]$ and $5 / 2^{-}[523]$ bands in ${ }^{161}$ Dy. The solid lines are linear fits to the data points, whereas the dashed lines connecting the only two available data points are included to guide the eye.

states. Although only fewer values are available for the decay of the $3 / 2^{-}$[521] band in ${ }^{161} \mathrm{Dy}$, also here a signature dependence is observed. In the case of the decay of the $5 / 2^{-}$[523] band in ${ }^{161} \mathrm{Dy}$, interestingly the splitting is strongly quenched as compared to the former case (compare Fig. 9). Looking back to Fig. 8, there seems to be a clear correspondence between the magnitude of the energy and the $B(E 1) / B(E 2)$ signature splittings. Whereas the $3 / 2^{-}[521]$ band shows an energy splitting of up to $60 \mathrm{keV}$ in the spin range, in which clearly signature dependent $B(E 1) / B(E 2)$ were obtained, it is only around $15 \mathrm{keV}$ for the $5 / 2^{-}$[523] band.

It is tempting to attribute the observed signature dependence of the $B(E 1) / B(E 2)$ ratios for the $3 / 2^{-}[521]$ bands to a signature dependence of the $B(E 1)$ rather than the $B(E 2)$ values as a $30 \%$ difference between the $Q_{0}$ moments of the two signature partners of the $3 / 2^{-}$band seems rather unlikely in view of the stable deformation of the neutron-rich Dy nuclei. In this context, it is also interesting to study whether the $B(M 1) / B(E 2)$ ratio for the $3 / 2^{-}$[521] band shows a signature dependence, too. For this purpose, we determined the experimental $B(M 1) / B(E 2)$ ratios from the intensity ratios using the formula

$$
\frac{B(M 1 ; I \rightarrow I-1)}{B(E 2 ; I \rightarrow I-2)}=0.697 \frac{E_{\gamma}^{5}(E 2)}{E_{\gamma}^{3}(M 1)} \frac{I_{\gamma}(M 1)}{I_{\gamma}(E 2)}\left(\frac{\mu_{N}}{e \mathrm{~b}}\right)^{2},
$$

assuming the $\Delta I=1$ transitions to be of pure $M 1$ nature. Since the mixing ratio for the $\Delta I=1$ transitions connecting the two signatures of the decay sequences are expected to be 


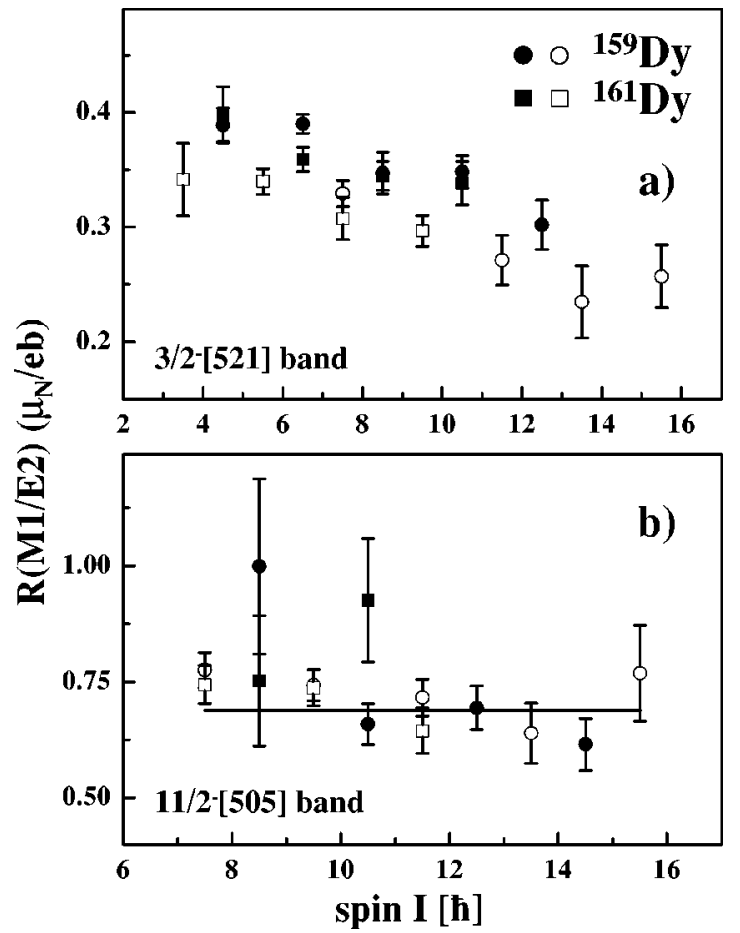

FIG. 10. Experimental $R(M 1 / E 2)$ ratios (see text for the definition) as a function of spin for (a) the $3 / 2^{-}$[521] bands and (b) the $11 / 2^{-}$[505] bands in ${ }^{159} \mathrm{Dy}$ and ${ }^{161} \mathrm{Dy}$. The solid line shows the theoretical prediction obtained in the strong-coupling model.

small, the uncertainty introduced by this assumption is likely small compared to the uncertainties in the determination of the experimental branching ratios. As in the case of the $B(E 1) / B(E 2)$ ratios discussed above, the experimental branching ratios have been determined from the intensities in individual coincidence spectra with gates on the respective feeding transitions. In anology to the discussion of the $B(E 1) / B(E 2)$ ratios, we define the quantity

$$
R(M 1 / E 2) \equiv\left[\frac{B(M 1 ; I, K \rightarrow I-1, K)}{B(E 2 ; I, K \rightarrow I-2, K)}\right]^{1 / 2} \mid \frac{\langle I, K, 2,0 \mid I-2, K\rangle}{\langle I, K, 1,0 \mid I-1, K\rangle},
$$

which is shown for the $3 / 2^{-}[521]$ bands in ${ }^{159}$ Dy and ${ }^{161}$ Dy in Fig. 10(a). Indeed, a signature dependence is observed for this quantity, too. As in the case of the $E 1$ transitions, also the $B(M 1) / B(E 2)$ ratio is larger for the decay of positive signature states. However, the signature splitting of the $R(M 1 / E 2)$ ratios is much smaller than the splitting observed for the $R(E 1 / E 2)$ ratios of the same band in ${ }^{159}$ Dy supporting our assumption that the $E 1 / E 2$ splitting is not due to a signature dependence of the quadrupole moment $Q_{0}$.

As discussed above, the new band identified to belong to ${ }^{161}$ Dy [compare Fig. 3(c)] seems to be very similar to the $11 / 2^{-}[505]$ band in ${ }^{159}$ Dy and we therefore tentatively assigned it to be based on the same neutron orbit. In both bands several strong $\Delta I=1$ cascade as well as crossover $E 2$ transitions are observed and both show a strong-coupling character. To further support our assignment, we compared the experimental $B(M 1 ; I \rightarrow I-1) / B(E 2 ; I \rightarrow I-2)$ ratios for the $11 / 2^{-}$[505] bands in ${ }^{159,161}$ Dy with each other and with the values obtained in the rotational model [25]. To determine theoretical $R(M 1 / E 2)$ ratios within the rotational model, the following formula has been used:

$$
R(M 1 / E 2)=\sqrt{\frac{12}{5}} \frac{K\left(g_{K}-g_{R}\right)}{Q_{0}} .
$$

For the parameters we used the values $K=11 / 2 \hbar, g_{K}-g_{R}$ $=-0.55$, and $Q_{0}=6.8 \mathrm{eb}$ (taken from Ref. [12]).

The resulting values for the $11 / 2^{-}[505]$ bands in ${ }^{159,161}$ Dy are shown as a function of spin in Fig. 10(b). The agreement between experimental and theoretical values is reasonably good thus supporting the assignment of the $11 / 2^{-}[505]$ configuration to the new band in ${ }^{161} \mathrm{Dy}$.

\section{PROJECTED SHELL MODEL CALCULATIONS}

The projected shell model (PSM) is a shell model approach that describes rotational bands in heavy nuclei. The PSM builds its shell model basis by selecting configurations from the deformed single-particle states and performing angular momentum projection exactly. It allows us to treat heavy nuclei in a shell model framework because important nuclear correlations are easily taken into account in a manageable configuration space. In recent years, the PSM has become a useful tool to analyze the high-spin data, for both normally deformed and superdeformed nuclei. It has already been used by us to describe the yrast lines and excited bands of the even-even ${ }^{160,162}$ Dy isotopes in Ref. [7]. In this work, PSM calculations of rotational bands of both parities in the odd isotopes ${ }^{159,161,163}$ Dy will be presented.

We will give only a brief reminder of the PSM here, more details can be found elsewhere [26]. The ansatz for the wave function is given by

$$
|\sigma, I M\rangle=\sum_{\kappa} f_{\kappa}^{\sigma} \hat{P}_{M K_{\kappa}}^{I}\left|\phi_{\kappa}\right\rangle
$$

The index $\sigma$ labels the states with the same angular momentum and $\kappa$ the basis states having quantum number $K$. The operator $\hat{P}_{M K}^{I}$ projects the quantum numbers $I$ and $M$ and generates states of good angular momentum, thus restoring the rotational symmetry violated in the deformed mean field. The $f_{\kappa}^{\sigma}$ are the weights of the basis states $\left|\phi_{\kappa}\right\rangle$ to be determined by the variational principle which in this case leads to the Hill-Wheeler equation. In the PSM the basis states $\left|\phi_{\kappa}\right\rangle$ are multiquasiparticle states of the vacuum $|\Phi\rangle$ determined by a Nilsson+BCS approach. The complexity of the model depends obviously on the basis states $\left|\phi_{\kappa}\right\rangle$ being considered. To describe the even-even Dysprosium isotopes we have used a basis spanned by the quasiparticle vacuum $|\Phi\rangle$, twoquasiproton states, two-quasineutron states, and fourquasiparticle states (two-quasineutron and two-quasiproton) [7]. This basis was large enough to consider neutron and proton alignments simultaneously. The current version of the odd- $A$ (in our case odd- $N$ ) code [27], however, only handles so far a limited basis of the type 


$$
\left\{\alpha_{n_{k}}^{\dagger}|\Phi\rangle, \quad \alpha_{i}^{\dagger} \alpha_{j}^{\dagger} \alpha_{n_{k}}^{\dagger}|\Phi\rangle\right\} .
$$

While the index $n_{k}$ is the one-quasiparticle neutron state that can be taken from either positive or negative parity orbitals, $(i, j)$ is limited to two-quasiparticle proton or twoquasiparticle neutron states which, together with the onequasiparticle neutron state, build the three-quasiparticle states. That means that configurations involving both twoproton and two-neutron states are neglected in the code. This approach should cause no problem as long as one describes those rotational bands with the alignment effect caused mainly by one kind of pairs (either neutron or proton). In fact, the basis of Eq. (8) was found to be appropriate in the description of the rotational alignment for many $i_{13 / 2}$ bands in odd- $N$ nuclei with the rotational alignment caused mainly by breaking the proton $h_{11 / 2}$ pairs [28]. As we shall see, however, for the structure of the negative parity bands studied in this paper the $N=5$ neutron orbitals are of importance. That means that for these bands, both $i_{13 / 2}$ neutron and $h_{11 / 2}$ proton orbitals are not blocked, and therefore a simultaneous alignment of neutron and proton pairs is possible.

The Hamiltonian used in the calculation is the pairing (monopole and quadrupole) plus quadrupole one. The quadrupole strength constant has been chosen to reproduce the quadrupole deformation parameter $\epsilon_{2}$ which takes the values 0.250 for ${ }^{159} \mathrm{Dy}, 0.255$ for ${ }^{161} \mathrm{Dy}$, and 0.270 for ${ }^{163} \mathrm{Dy}$. The monopole-pairing-force constants for neutrons $(-\operatorname{sign})$ and protons $(+$ sign $)$ are usually taken as $\left(G_{M}=[20.12\right.$ $\left.\mp 13.13(N-Z) / A] A^{-1}\right)$ in the rare-earth calculations [26], the values being adjusted to roughly reproduce the known energy gaps. In the present calculations for odd- $N$ systems, we found that the standard choice of the pairing force is a little too strong. Thus the monopole-pairing-force constants are slightly reduced by multiplying a factor of 0.94 in the present work. Finally, the strength parameter $G_{Q}$ for the quadrupole pairing is taken as $0.16 G_{M}$ for all three nuclei. No additional parameter adjustment is allowed for individual bands in any nucleus. In the present calculations, we have included active particles from three oscillator shells, $N$ $=3,4,5$ for protons and $N=4,5,6$ for neutrons.

The positive parity bands of the ${ }^{159,161,163}$ Dy nuclei are rather well described in the limited basis $\left\{\alpha_{n_{k}}^{\dagger}|\Phi\rangle, \alpha_{p_{i}}^{\dagger} \alpha_{p_{j}}^{\dagger} \alpha_{n_{k}}^{\dagger}|\Phi\rangle\right\}$, where only proton pairs $\left(p_{i}, p_{j}\right)$ from the $h_{11 / 2}$ orbital are included in the three-quasiparticle configurations. The physical reason behind this is that the blocked neutron orbital, the $i_{13 / 2}$ one, is inhibiting a neutron alignment such that only the high- $j$ proton orbitals are significantly contributing to the generation of high angular momenta. Our model space thus contains the most important configurations, determining the structure of the positive parity bands. In the left column of Fig. 11 the calculated alignments $i(\omega)$ of the $5 / 2^{+}[642]$ bands are compared to the experimental ones for ${ }^{159,161,163} \mathrm{Dy}$. The Harris parameters used to deduce the theoretical and experimental alignments are the same as those used in Fig. 7. We find that the theory provides the gross features in all three isotopes although some differences are present. In particular the alignment calculated for ${ }^{159} \mathrm{Dy}$ is larger than that in the experiment for
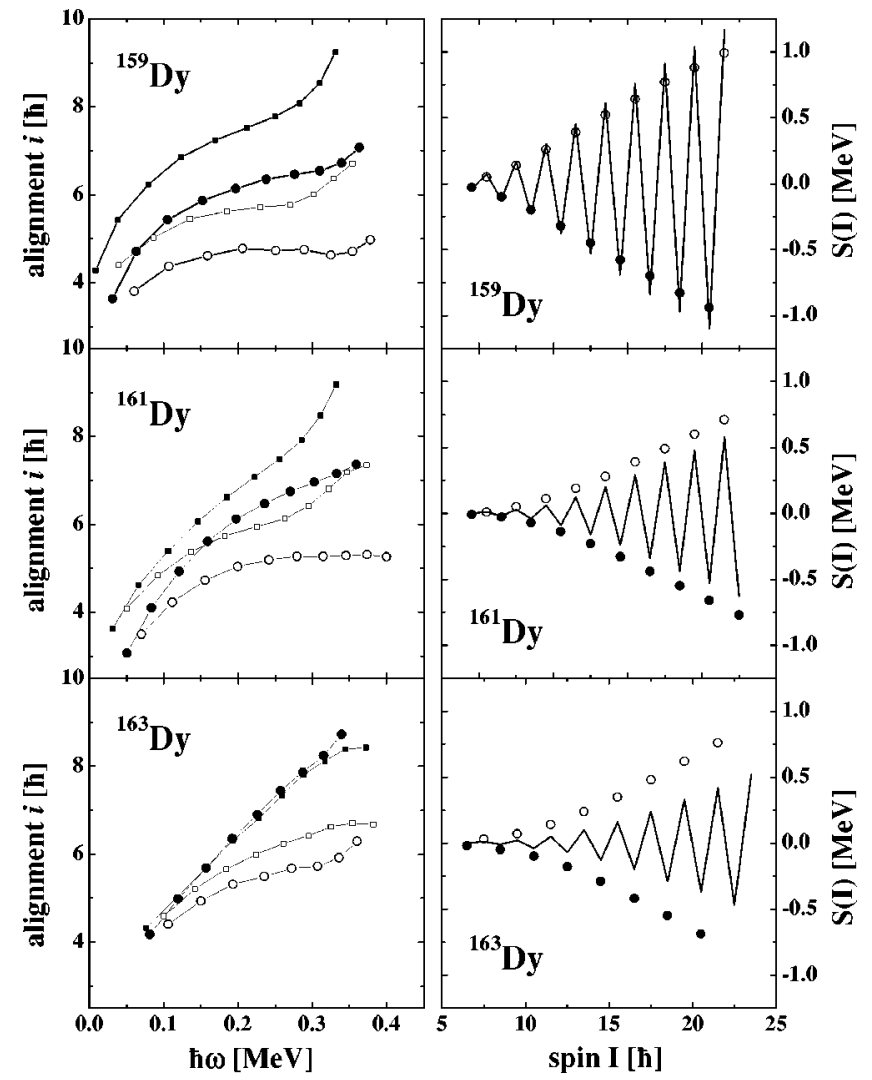

FIG. 11. Comparison between experimental and PSM alignment curves (left) and energy differences $S(I)$ (right) for the positive parity $5 / 2^{+}$[642] bands in ${ }^{159,161,163}$ Dy. $\alpha=+1 / 2(\alpha=-1 / 2)$ states are shown as filled (open) symbols. The experimental values are shown as circles, the results of the TQPM calculations as squares with lines (left) and solid lines (right).

both signatures. With increasing neutron number the positive signature branch gets closer to the experiment. This plot provides separate information for the positive and negative signature bands. The relative separation between the levels of the signature partner bands is provided by $S(I)$ which is represented on the right hand side of Fig. 11. We get good agreement with the data for ${ }^{159}$ Dy and ${ }^{161}$ Dy and a smaller splitting than experimentally observed for ${ }^{163} \mathrm{Dy}$.

For the negative parity bands, the blocked neutron orbital is not an $i_{13 / 2}$ orbital and the alignment of a neutron $i_{13 / 2}$ pair may thus compete with that of high- $j$ proton pairs. To see this effect clearly, we have performed two separate calculations. In the first calculation the basis states $\left\{\alpha_{n_{k}}^{\dagger}|\Phi\rangle, \alpha_{p_{i}}^{\dagger} \alpha_{p_{j}}^{\dagger} \alpha_{n_{k}}^{\dagger}|\Phi\rangle\right\}$ have been used. This is the same configuration space used in the previous calculations for the positive parity bands. The quasiparticle index $\left(p_{i}, p_{j}\right)$ in the three-quasiparticle states represents a proton pair from the $h_{11 / 2}$ orbital. In the second calculation, we used $\left\{\alpha_{n_{k}}^{\dagger}|\Phi\rangle, \alpha_{n_{i}}^{\dagger} \alpha_{n_{j}}^{\dagger} \alpha_{n_{k}}^{\dagger}|\Phi\rangle\right\}$ in an obvious notation. We shall refer to TQPM (TQNM) for the first (second) configuration space, meaning that only two quasiproton (quasineutron) mixing is allowed. We are aware that in order to describe a simultaneous proton and neutron alignment in odd- $A$ systems properly one should include five quasiparticle states, 


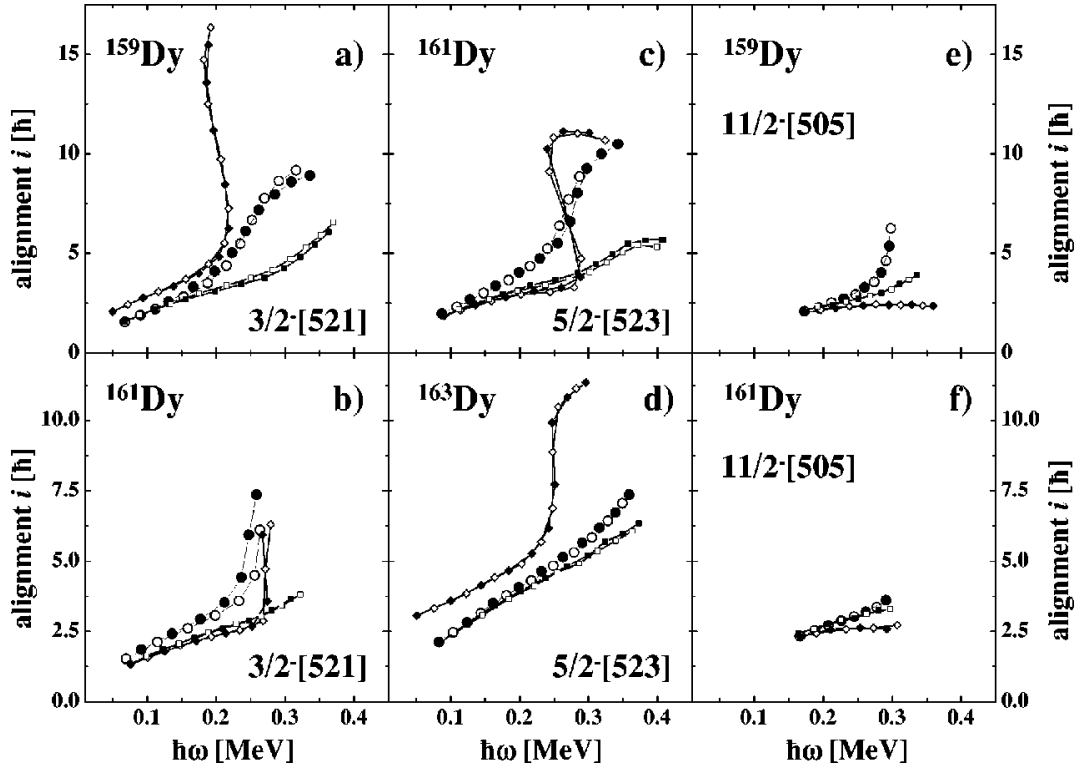

FIG. 12. Comparison between experimental and PSM alignment curves for the negative parity bands in ${ }^{159,161,163} \mathrm{Dy}$. (a) $3 / 2^{-}[521]$ band in ${ }^{159} \mathrm{Dy}, \quad$ (b) $3 / 2^{-}[521]$ band in ${ }^{161} \mathrm{Dy}$, (c) $5 / 2^{-}$[523] band in ${ }^{161} \mathrm{Dy}$, (d) $5 / 2^{-}$[523] band in ${ }^{163} \mathrm{Dy}$, (e) $11 / 2^{-}$[505] band in ${ }^{159} \mathrm{Dy}$, and (f) $11 / 2^{-}$[505] band in ${ }^{161}$ Dy. Experimental values are given as filled (open) circles for the $\alpha=+$ $(\alpha=-)$ signature. The results from the PSM calculation allowing for the alignment of a $h_{11 / 2}$ proton pair (TQPM) are shown as squares with lines and the ones from the calculations considering an $i_{13 / 2}$ neutron alignment (TQNM) as rhombuses with lines. which is numerically quite involved and out of scope of our present PSM code.

In Fig. 12 the alignment plots for the negative parity bands are shown for the three Dy isotopes. These calculations based on a limited model space, though not very successfull in providing a quantitative agreement with all the experimental data, do have the advantage of indicating where proton or neutron alignment is taking place. In panel (a) the results of these calculations together with the data are shown for the $3 / 2^{-}[521]$ band in ${ }^{159} \mathrm{Dy}$. Up to $\hbar \omega \approx 0.2 \mathrm{MeV}$ the TQPM solution provides a good agreement with the experiment, i.e., no additional neutron alignment is required. For $\hbar \omega>0.2 \mathrm{MeV}$, however, the alignment provided by the TQPM solution is not sufficient to reproduce the data. As we can see in the TQNM results we get enough alignment from the neutron system, indicating that neutron alignment takes place for $\hbar \omega>0.2 \mathrm{MeV}$. For the same band in ${ }^{161} \mathrm{Dy}$, we see in panel (b) that the alignment that takes place in the data for $\hbar \omega \approx 0.25 \mathrm{MeV}$ is also due to neutron alignment. For the $5 / 2^{-}$[523] band in ${ }^{163}$ Dy [panel (d)] we find that the TQPM calculation gives good agreement with the data and that only at high angular frequencies a small neutron alignment may ocurr. This is not the case for the same band in ${ }^{161} \mathrm{Dy}$ [panel (c)], where we find that both the proton and neutron contributions are needed already for small angular frequency. Furthermore, a strong neutron alignment takes place around $\hbar \omega>0.25 \mathrm{MeV}$. Finally for the band $11 / 2^{-}[505]$ in ${ }^{161}$ Dy [panel (f)], we find good agreement with the data for the TQPM solution, while the alignment observed for the same band in ${ }^{159}$ Dy [panel (e)] requires more alignment than indicated by the TQNM and TQPM calculations.

\section{CONCLUSIONS}

High-spin states in the odd ${ }^{159,161,163}$ Dy isotopes have been studied via the incomplete fusion reactions ${ }^{158,160} \mathrm{Gd}\left({ }^{7} \mathrm{Li},(p, d, t) x n\right)$. In each of the nuclei, known rotational bands have been extented to higher spins and a new band has been observed in ${ }^{161} \mathrm{Dy}$. Based on a comparison with a similar band in ${ }^{159} \mathrm{Dy}$, we suggest that the new band in ${ }^{161}$ Dy is built on the $11 / 2^{-}$[505] neutron single-particle orbit. For these $11 / 2^{-}$[505] bands in both nuclei ${ }^{159,161}$ Dy, the $B(M 1) / B(E 2)$ ratios have been compared with theoretical values obtained within the strong-coupling model and good agreement has been found. Eleven (four) $E 1$ transitions from both signatures of the neutron $3 / 2^{-}$[521] band to the $5 / 2^{+}[642]$ band have been observed in ${ }^{159}$ Dy $\left({ }^{161} \mathrm{Dy}\right)$ as well as further $14 E 1$ transitions between the $5 / 2^{-}$[523] and the $5 / 2^{+}[642]$ bands in ${ }^{161} \mathrm{Dy}$. Whereas no signature dependence has been observed in the decay of the $5 / 2^{-}$[523] band in ${ }^{161} \mathrm{Dy}$, the measured $B(E 1) / B(E 2)$ ratios for the decay of states with positive signature are systematically larger than the ones for the decay of negative signature states in the case of the $3 / 2^{-}[521]$ band in ${ }^{159} \mathrm{Dy}$. For the latter band, also a signature dependence is observed in the $B(M 1) / B(E 2)$ ratios and a correlation between the observed signature dependence of the electromagnetic decay properties and the energy staggering of the bands has been discussed. Finally, the properties of all observed rotational bands, in particular the band crossing frequencies and alignments, have been discussed and compared to projected shell model calculations. Whereas the agreement between experiment and theory is good for the positive parity bands built on the $5 / 2^{+}[642] i_{13 / 2}$ neutron orbit, it is not satisfactory for the negative parity bands due to limitations of the computer codes used in the present work.

\section{ACKNOWLEDGMENTS}

We are most grateful to the crew of the tandem accelerator at the LNL Legnaro for the friendly and efficient cooperation. We thank J. L. Egido for many fruitful discussions. This work was supported by the Deutsches Bundesministerium für Bildung, Wissenschaft, Forschung und Technologie (BMBF). A.J. acknowledges support by the Deutsche Forschungsgemeinschaft (DFG) within the Heisenberg program. 
[1] A. Johnson, H. Ryde, and J. Sztarkier, Phys. Lett. 34B, 605 (1971)

[2] E. Grosse et al., Phys. Rev. Lett. 31, 840 (1973); 32, 74 (1974).

[3] L. L. Riedinger et al., Phys. Rev. Lett. 33, 1346 (1974).

[4] I. Hamamoto et al., Phys. Lett. B 226, 17 (1989).

[5] I. Hamamoto, Nucl. Phys. A557, 515c (1993).

[6] G. B. Hagemann et al., Phys. Rev. C 47, 2008 (1993).

[7] A. Jungclaus et al., Phys. Rev. C 66, 014312 (2002).

[8] H. Beuscher et al., Nucl. Phys. A249, 379 (1975).

[9] D. R. Haenni et al., Bull. Am. Phys. Soc. 29, 1043, CC14 (1984).

[10] W. Andrejtscheff et al., Nucl. Phys. A225, 300 (1974).

[11] X. Liang et al., Eur. Phys. J. A 10, 41 (2001).

[12] M. Sugawara et al., Nucl. Phys. A699, 450 (2002).

[13] S. A. Hjorth et al., Nucl. Phys. A184, 113 (1972).

[14] V. A. Bondarenko et al., Proceedings of the 33rd Annual Con- ference on Nuclear Spectroscopic Structures of Atomic Nuclei, Moscow, 1983.

[15] T. W. Conlon et al., Nucl. Phys. A104, 213 (1967).

[16] E. Minehara et al., Phys. Rev. C 35, 858 (1987).

[17] H. H. Schmidt et al., Nucl. Phys. A504, 1 (1989).

[18] J. D. Garrett et al., Phys. Rev. Lett. 47, 75 (1981).

[19] J. Kownacki et al., Nucl. Phys. A394, 269 (1983).

[20] A. Fitzpatrick et al., Nucl. Phys. A582, 335 (1995).

[21] W. B. Gao et al., Phys. Rev. C 44, 1380 (1991).

[22] G. B. Hagemann et al., Nucl. Phys. A618, 199 (1997).

[23] H. J. Jensen et al., Nucl. Phys. A695, 3 (2001).

[24] Y. R. Shimizu et al., Nucl. Phys. A611, 22 (1996).

[25] A. Bohr and B. R. Mottelson, Nuclear Structure, (Benjamin, New York, 1969), Vol. II.

[26] K. Hara and Y. Sun, Int. J. Mod. Phys. E 4, 637 (1995).

[27] Y. Sun and K. Hara, Comput. Phys. Commun. 104, 245 (1997).

[28] K. Hara and Y. Sun, Nucl. Phys. A537, 77 (1992). 